Rhode Island College

Digital Commons @ RIC

\title{
A Computer-Based Stroke Education Orientation Program for Nurses on a Designated Stroke Unit: A Program Development, Quality Improvement Project
}

Melanie Marco

Rhode Island College

Follow this and additional works at: https://digitalcommons.ric.edu/etd

Part of the Cardiovascular Diseases Commons, and the Nursing Commons

\section{Recommended Citation}

Marco, Melanie, "A Computer-Based Stroke Education Orientation Program for Nurses on a Designated Stroke Unit: A Program Development, Quality Improvement Project" (2017). Master's Theses, Dissertations, Graduate Research and Major Papers Overview. 181.

https://digitalcommons.ric.edu/etd/181

This Thesis is brought to you for free and open access by the Master's Theses, Dissertations, Graduate Research and Major Papers at Digital Commons @ RIC. It has been accepted for inclusion in Master's Theses, Dissertations, Graduate Research and Major Papers Overview by an authorized administrator of Digital Commons @ RIC. For more information, please contact digitalcommons@ric.edu. 
A COMPUTER-BASED STROKE EDUCATION ORIENTATION PROGRAM FOR NURSES ON A DESIGNATED STROKE UNIT:

A PROGRAM DEVELOPMENT, QUALITY IMPROVEMENT PROJECT

by

Melanie Marco

A Major Paper Submitted in Partial Fulfillment of the Requirements for the Degree of

Master of Science in Nursing

in

The School of Nursing

Rhode Island College

2017 


\begin{abstract}
Six million people in the United States live with disabilities due to stroke (AHA, 2016). Great progress has been made over the past 50 years with the introduction of medications to reduce disability from stroke. Fibrinolytic therapy is the treatment of choice for ischemic stroke and significantly decreases rate of death or disability by $30 \%$ (Jauch et al., 2013).
\end{abstract}

Use of fibrinolytic therapy for ischemic stroke may increase if patients sought treatment immediately. As a result of years of research, the AHA and The Joint Commission, identified eight core measures and best practice guidelines to improve care and for stroke patients. Hospitals that meet these evidence-based practice standards are designated Primary Stroke Centers (PSC).

A needs assessment, employing the W. K. Kellogg Logic Model, determined that an up-dated, comprehensive stroke orientation education program was needed to maintain Primary Stroke Center designation. Primary Stroke Centers designated by The Joint Commission show better patient outcomes. Highly trained clinicians are imperative to care for such complex patients. The purpose of this quality improvement project was to develop a comprehensive, evidence-based, four-module, on-line orientation curriculum for nurses who work on a stroke-designated unit in a Primary Stroke Center. 


\section{Table of Contents}

Background/Statement of the Problem ................................................................... 1

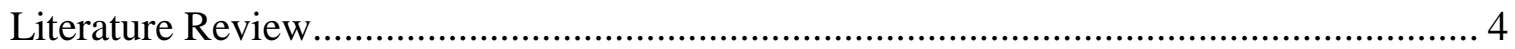

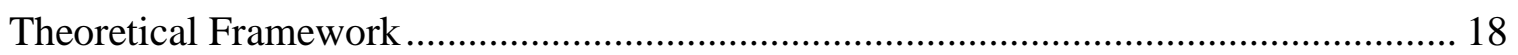

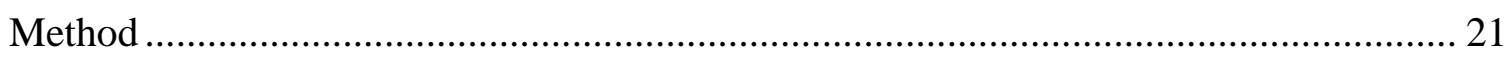

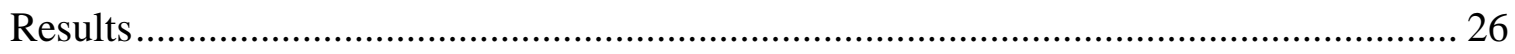

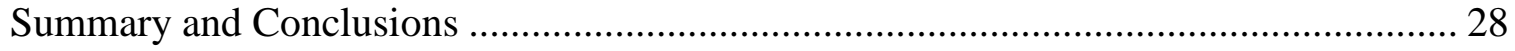

Recommendations and Implications for Advanced Nursing Practice ........................... 31

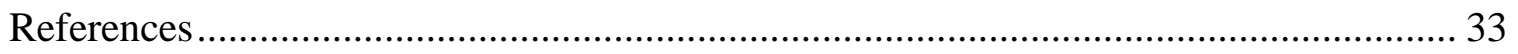

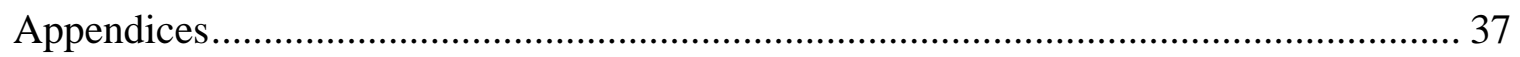


A Computer-Based Stroke Education Orientation Program for Nurses on a

Designated Stroke Unit:

A Program Development, Quality Improvement Project

\section{Background/Statement of the Problem}

Stroke is a devastating disease and the leading cause of disability in adults.

According to the American Heart Association (AHA), 1 in 20 deaths in the United States are a result of a stroke. Someone dies from a stroke every four minutes (AHA, 2016). Currently, there are over six million people living with disabilities as a result of stroke (AHA, 2016). Timely thrombolysis therapy is imperative to improve embolic stroke outcomes (Goyal et al., 2016).

Since 2008, stroke has dropped from being the third leading cause of death to the fourth leading cause of death in the Unites States. This may be in part due to the AHA's dedication to bringing increased awareness about stroke to the public (Jauch et al., 2013). Since 1949, the AHA has funded $\$ 3.1$ billion toward cardiovascular and stroke research (Schwamm et al., 2010). The AHA's stated mission is “to build healthier lives, free of cardiovascular disease and stroke" (AHA, 2016). Consistent with their mission, the AHA formed the American Stroke Association (ASA) division in 1998. Before the formation of the ASA, an American was having a stroke every 53 seconds and dying of a stroke every 3.3 minutes. The ASA set a goal to reduce stroke deaths $25 \%$ by 2010 . They accomplished that goal in 2009 by focusing education on stroke prevention, diagnosis and treatment and incorporating these into clinical practice (Schwamm et al., 2010). The education campaign included audiovisual programs, lectures, printed material and television and billboard advertisements. The education was geared toward not only patients but also to their family members and caregivers (Jauch et al., 2013). The Joint 
Commission (TJC), along with the American Heart Association, developed eight core stroke core measures that were published in 2008. There is a correlation between meeting the core measures and decreasing disability and mortality due to stroke. The core measures include treatment of conditions that lead to stroke, early stroke intervention and post-stroke treatment.

In 2003, TJC established a process for Primary Stroke Center (PSC) certification. Primary Stroke Center Certification recognizes hospitals that meet standards that support better outcomes for stroke care. To attain PSC certification, a hospital must have a dedicated stroke program which publicly reports outcomes and demonstrates evidence of continuous improvement. To meet these standards, a hospital must have a designated inpatient stroke care unit with highly qualified clinicians. The PSC must follow the AHA/ASA national guidelines, including acute care inpatient treatment through postdischarge care. A standard of care by qualified staff trained in stroke care must be demonstrated. Individualized care that meets the patient's needs and demonstrates patient involvement in his or her own care is also a requirement. Hospitals must collect stroke treatment performance data and use that data to continually assess and improve quality of care for stroke patients (TJC, 2016).

Stroke is a complex disease that affects multiple body systems requiring highly trained and skilled multidisciplinary team. Education for staff nurses working with stroke patients, especially on the dedicated inpatient stroke unit, is an essential component of care to improve outcomes for individuals. Nurses must be able to recognize signs of deteriorating neurological symptoms since $25 \%$ of patients exhibit worsening symptoms within the first 24 to 48 hours after stroke. A 2013 observational study demonstrated that 
patients admitted to a PSC had a $10.1 \%$ mortality rate compared to a $12.5 \%$ mortality rate for those admitted to a non-certified hospital. The same study showed a $3 \%$ increase in the use of fibrinolytic therapy in patients admitted to a PSC (Jauch et al., 2013).

Evidence of improved outcomes for patients admitted to a dedicated stroke unit supports the need for specialized stroke education and protocols. The Joint Commission now requires all stroke patients be admitted to a PSC and receive care on a designated stroke unit. Care on the stroke unit is focused on preventing further debilitation, deterioration and reducing medical complications. Nurses working at a primary stroke center are required by TJC to have initial orientation stroke education and annual competency review to ensure stroke patients receive high quality, current care. The Joint Commission also requires core stroke team members receive at least eight hours of education annually, as determined by the stroke program manager. The stroke program manager determines composition of the stroke team in collaboration with hospital leadership and other stakeholders (Daniels, Johnson \& Mackovjak, 2011). It is the responsibility of each primary stroke center to develop an education program for clinical staff.

The purpose of this quality improvement project was to develop a computer-based stroke education orientation program for nurses on a recently designated stroke unit within a PSC facility.

Next a review of literature will be presented. 


\section{Literature Review}

A comprehensive online search was completed using the databases CINAHL and PubMed. Searches were limited to 1999 to 2016. Keywords used in the search criteria were stroke, designated stroke units, education for nurses in primary stroke centers, core measures, and "get with the guidelines".

\section{History Stroke}

Stroke care in the early 1900 s was focused on treatment instead of prevention. In 1946 strokes were considered a chronic, terminal disease (Feldman, 1951). With medical advances and increased interest, care began to focus on prevention. The AHA funds studies related to basic, clinical and population science and since 1949 the AHA has funded \$3.1 billion in cardiovascular and stroke research (Schwamm et al., 2010). The AHA conducts their research by using an approach entitled: A Strategically Focused Research Network (SFRN). Strategically Focused Research Networks are comprised of three to four facilities or centers, working on three projects each that are focused on one strategic area. The research milestones reached by AHA from 1950 to 1970 include research that first linked dietary fat with cholesterol that is directly related to stroke risk. The research also demonstrated that chlorothiazide reduces blood pressure and high blood pressure is associated with decreased life expectancy. These studies influenced and guided the shift of stroke care to a focus on prevention. A cross-sectional, populationbased survey collected over 20 years revealed that as awareness, treatment and control of hypertension increased, blood pressure decreased. In addition, as awareness, treatment 
and control of hypercholesterolemia increased, total cholesterol levels decreased. Finally, due to anti-smoking campaigns, smoking rates decreased (Towfighi \& Saver, 2011).

The Framingham Heart Study is a longitudinal research study that started in 1948 when a group of scientists embarked on a journey to determine risks for cardiac disease. At the time there was little known about cardiac, stroke risk and stroke disease. The study followed participants who had not yet developed any cardiac disease. Participants who were 55 years old were followed for up to 50 years over 3 consecutive periods (1950 to 1977; 1978 to 1989 ; and 1990 to 2004). The findings revealed that the incidence of stroke per 1000 person-years in each of the three periods was 7.6, 6.2, and 5.3 respectively. The lifetime risk at age 65 years decreased from $19.5 \%$ to $14.5 \%$ in men and $18.0 \%$ to $16.1 \%$ in women. Among stroke survivors in the Framingham Heart Study, all had a decrease in systolic blood pressure, cholesterol levels and incidence of hypertension. The proportion of participants receiving treatment for hypertension, and the number of participants who smoked decreased also (Towfighi \& Saver, 2011).

This early, groundbreaking research and subsequent advances in research led to the development of core measures that primary stroke centers must follow when treating stroke patients. The core measures are rigorously studied and verified to determine clinical reliability, validity and align with the most current evidence based practice. They were developed collaboratively with contributions from health care professionals, providers, expert in the related field, government agencies including Centers for Medicare \& Medicaid Services, American Heart Association (AHA) and employers (TJC, 2016). 
Prior to 2008, the public was advised to seek treatment based on if they experienced one or more of the following symptoms: sudden weakness: sudden slurred speech: sudden headache: sudden dizziness: and sudden visual loss. These symptoms were referred to as the "5 Suddens" of stroke and were widely used among health care providers to identify patients at risk for stroke. In 2008, the AHA national guidelines changed to the FAST (face, arm, speech, time) acronym to define symptoms of stroke. One or more of face weakness, arm weakness, and speech difficulty symptoms are present in $88 \%$ of all strokes. One hundred percent of individuals, educated on the FAST acronym, remembered that facial droop and slurred speech are stroke warning signs. Ninety-eight percent were able to remember that arm weakness or numbness was also a sign of stroke. The AHA promoted the message to the public to call 9-1-1 immediately when a stroke is suspected (AHA, 2016). The recent National Hospital Ambulatory Medical Care Survey (NHAMCS) showed that only 53\% of stroke patients used EMS. Therefore, in 2008 the "FAST" education campaign was launched by the American Heart Association (Jauch et al., 2013).

Today a stroke is defined as rapid onset of neurological deficits due to an interruption in blood supply to the brain. (Lindley, 2008). Another definition of a stroke is "a clinical syndrome characterized by rapidly developing focal symptoms or with loss of cerebral function lasting for 24 hours" (Goldszmidt \& Caplan, 2010).

\section{Stroke Physiology}

The brain is large and complex and is divided into lobes with each lobe controls certain functions. The brain receives its energy from oxygen via blood supply through 
cerebral arteries and is dependent on this energy for hemodynamic stability. Without the blood supply brain tissue is damaged and cell death occurs in areas of the brain deprived of oxygen. Deprivation of oxygen for one minute leads to irreversible damage. Focal symptoms are weakness affecting one side of the body or a loss of vision. Strokes interrupt blood supply to an area of the brain and are classified into two major types, ischemic and hemorrhagic (Eigsti, \& Henke, 2006). Distinguishing between the two types influences treatment.

Ischemic Stroke. Ischemic strokes are the most common type of stroke, comprising $80 \%$ of all strokes and are caused by an occlusion of the extracranial cerebral arteries, depriving the brain of oxygen (Caplan, 2016). Ischemic strokes are divided into two types: embolic and thrombotic. An embolism may be the result of a blood clot, plaque fragment, lipids or air that enters the circulation system and occludes a blood vessel. An embolism originates from another part of the body and travels, lodging in an artery in the brain, depriving it of circulation and oxygen. This in turn effects function controlled by that area of the brain. A thrombosis is an obstruction of the cerebral artery wall due to disease such as atherosclerosis, which is a thickening of the artery walls. A thrombus develops in the site of the occlusion and does not travel. The thickening occurs when the inner lining of the arteries is damaged and cellular waste accumulates in the artery due to the inner protective lining being damaged. When an artery in the brain is occluded, neurological symptoms result from oxygen deprivation and present as a focal neurological deficit. A focal neurological deficit occurs at the exact location in the brain associated with a specific abnormality. For example, left side weakness or problems 
swallowing is caused by an occlusion in the cerebral circulation of the brain where these functions are controlled (Caplan).

Hemorrhagic stroke. A hemorrhagic stroke occurs when an area of the cerebral arterial system becomes weak over time due to long-term or acute hypertension or vascular malformation. The weakened area can evolve into a pouch (aneurysm) and rupture causing bleeding into the brain (Davis \& Lockhart, 2016). Intracerebral hemorrhages cause blood to pool into the brain, which increases pressure on brain structures. The pressure then occludes blood flow to areas of the brain. A subdural hemorrhage may develop rapidly or slowly over a period of time. The blood, trapped between layers covering the brain, develops into a clot and compresses brain tissue causing focal neurological deficits. Bleeding into the space between the two outer membranes surrounding the brain causes subarachnoid hemorrhage. High blood pressure is a significant risk factor for this type of hemorrhage (Davis \& Lockhart).

\section{Risk Factors and Prevention}

There are modifiable and non-modifiable risk factors that contribute to stroke. Non-modifiable risk factors include being older then 45 , female gender, heredity, and Black, Hispanic or Asian ethnicity. Modifiable risk factors include smoking, atrial fibrillation, sleep apnea, hypertension, and history of substance abuse, history of TIA and previous stroke or MI. The AHA began the "Life's Simple 7" campaign in 2000. Life's Simple 7 are seven health prevention aspects that everyone can do to prevent heart disease and other cardiac diseases. The seven lifestyle changes are controlling cholesterol, stay active, eat better, stop smoking, reducing blood sugar, managing blood 
pressure and losing weight. These are essential life-style changes that every patient should be educated about (AHA, 2016).

The National Stroke Association (NSA) identifies three crucial steps in stroke prevention: identify risk; change risk factors; and ability to recognize and respond to stroke symptoms. The NSA recommends making lifestyle change that include eating a low fat diet, exercise, smoking cessation and limited alcohol intake (2016).

\section{Early Detection and Diagnostics}

It is vital that stroke symptoms are recognized and acted upon as soon as they are present. Many patients postpone treatment after they experience symptoms. Fibrinolytic therapy is the only approved therapy for ischemic stroke yet the use of fibrinolytic therapy within the recommended 60-minute time limit is low. The California Acute Stroke Pilot Registry (CASPR) was developed in California in an effort to collect data regarding stroke outcomes (Jauch et al., 2013). Data collected on 374 patients showed that fewer than half of patients sought treatment within one hour of symptom onset and in addition $50 \%$ of patients did not know that their symptoms may be indicative of a stroke. The California Acute Stroke Pilot Registry reported that administration of fibrinolytic treatment could increase to $28 \%$ if patients sought treatment as soon as symptoms began. Since neurological outcomes are strongly dependent on how quickly treatment is started, it is essential that time-sensitive interventions begin as soon as possible (California Acute Stroke Pilot Registry (CASPR) Investigators, 2005). Every minute that treatment is delayed equals millions of dead brain cells so it is vital for patients, caregivers and healthcare providers to recognize symptoms and seek treatment immediately (Jauch). 
The AHA (2016) recommends teaching the public the mnemonic FAST. The F stands for facial drooping, A for arm or leg weakness, $\mathrm{S}$ for speech slurred and $\mathrm{T}$ for time to call 911/code stroke. The AHA uses the slogans "Time is Brain" and "The Golden Hour" to emphasize that time to treatment is vital to outcomes (2016). The "Golden Hour" is the concept, used in emergency medicine, that is applied to conditions when timed interventions are vital to outcome. The "Golden Hour" for stroke was developed in 19-- (Saver et al, 2010). In the acute stroke setting, the "Golden Hour" begins at the time the patient arrives to the Emergency Department or, for the inpatient, the time the code stroke is initiated. The patient must be seen by the provider within 10 minutes and evaluated by the stroke team within 15 minutes. A CT scan of the brain must be completed within 25 minutes with results reported within 45 minutes and available to stroke team to make treatment decisions. Fibrinolytic therapy must be initiated within 60 minutes of arrival to the ED unless contraindicated (AHA, 2016).

In a study looking at April 2003 to September 2009, researchers examined stroke patient records from 1082 hospital emergency rooms. Data from over 25,505 stroke patients was abstracted and revealed that only 6790 patients eligible for thrombolytic therapy received the therapy within one hour of arrival to emergency room. These findings supported the fact that AHA needed to focus on the redesign of hospital structures to improve patient outcomes (Fonarow et al., 2011).

Many factors influence the success of the Golden Hour. There are interventions that have to be completed simultaneously within that Golden Hour. It is up to each Primary Center to ensure completion of each time sensitive intervention. If it is 
determined by CT scan that the stroke is ischemic, then a thrombolytic agent may be given. Immediate intervention to remove the occlusion and reperfuse the brain tissue will preserve brain tissue and symptoms will improve (Elkind, 2009). Tissue-Plasminogen Activator (tPA), first approved by the FDA 20 years ago, is the thrombolytic given to stroke patients. In order to be considered as a tPA candidate, certain criteria must be met. Inclusion criteria includes: less than four and one-half hours from stroke onset if last known well (LKW) is known;; no cerebral hemorrhage as evidenced by CT scan; early infract not greater than 1/3 of Middle Cerebral Artery (MCA) territory and National Institutes of Health Stroke Scale (NIHSS) score of 4-20 (Elkind, 2009). tPA activates plasminogen which breaks down fibrin and dissolves the clot. For maximum benefit tPA should be administered as soon as possible.

National Institute of Health Stroke Scale (NIHSS) must be completed by a certified clinician before fibrinolytic therapy may be administered. NIHSS is a standardized tool used by healthcare providers to objectively assess 15 aspects of stroke related symptoms. The 15 aspects are $\mathbf{1}$-level of consciousness, 1a.- responsiveness, $\mathbf{1 b}$ questions, 1c-commmands, 2-orientation, 3-response to commands, 4-gaze, 5-visual fields, 6-facial movement, 7-motor function, 8-limb ataxia, 9-sensory, 10-language, 11articulation, and 12-extinction. Each aspect is given a numeric value. The higher the NIHSS score, the larger the area of brain infarction.

In preparing the AHA guidelines for managing ischemic stroke a two-part National Institutes of Neurological Disorders and Stroke trial compared patients who received tPA within 3 hours of symptom onset. The trial consisted of 624 patients. 
Neurological outcomes were based on their based on their NIHSS 24 hours after treatment and then at 3 months after treatment. The trial showed that tPA treatment decreased disability from $40 \%$ to $28 \%$ and neurological deficits decreased from $34 \%$ to 20\% (Jauch et al., 2013).

\section{Primary Stroke Certification}

Primary Stroke Certification (PSC) was developed by TJC in 2000. The TJC defines a PSC as a facility that provides pre and post hospital care, as well as acute stroke care to patients. Primary Stroke Center contains standards that are organized into chapters that address five topics that include program management, delivering or facilitating clinical care, supporting self- management, clinical information management and performance improvement and measurement. All certified primary stroke centers have to follow quality measures and must report their performance in program management to TJC (TJC, 2016). With the introduction of organized stroke care especially Primary Stroke Centers in the United States; reduction in mortality among stroke admissions has been documented (Towfighi \& Saver, 2011).

Since TJC began certifying facilities over 800 facilities nationwide have been certified. Regardless if a facility participates in TJC certification they are still required to record stroke patient outcomes in a national database. In clustered, controlled clinical trials outcomes in certified PSC hospitals, that provided specialized stroke care, demonstrated better patient outcomes compared to non-certified hospitals. Numerous observational studies showed that PSC had higher compliance with "golden hour" which 
in turn shorten the door to needle (DTN) time. Primary Stroke Centers with organized stroke protocols proven to maintain these outcomes over time. (Jauch et al., 2013).

Rapid stroke recognition and appropriate priority of EMS dispatch strongly influences stroke outcomes. Therefore, the AHA worked closely with emergency management systems to standardize and strengthen pre-hospital protocols. The AHA performed a nationwide survey that verified the fact that stroke care by EMS had improved. AHA received responses from 5410 (35\%) of the 15,000 to 17,000 EMS agencies serving $91 \%$ of the United States population. The survey found that $74 \%$ of the assessment tools and $75 \%$ of the treatment protocols were used and were in alignment with AHA guidelines (Schwamm et al., 2010). In addition, an increasing number of states, including Rhode Island, require emergency medical services to transport stroke patients to stroke certified institutions only. Hospitals therefore have a financial incentive and a community responsibility to achieve and maintain certification (Jauch et al., 2013).

In 2003, TJC developed eight core measures that primary stroke centers must follow when treating stroke patients and began certifying hospitals in 2004. Joint Commission core measures were developed with contributions from health care professionals, experts in the field, government agencies including Centers for Medicare \& Medicaid Services, American Heart Association (AHA) and employers. New measures are added if they can impact patient safety or patient outcomes. The measures must align with federal laws and regulations and have the ability to be measured (TJC, 2016).

Core Measures. The TJC Stroke core measures are illustrated below. 
STK-1: venous thromboembolism prophylaxis. Stroke patients are at high risk for deep vein thrombosis (DVT) and pulmonary embolism (PE). Death due to pulmonary embolism occurs in approximately $10 \%$ of stroke patients. DVT and PE are most likely to happen in the first three weeks after stroke. PE develops from venous thrombi in a paralyzed limb secondary to stroke. These can lead to life threatening complications. Early mobilization, external compression devices and use of antithrombotic agents are interventions that meet the requirement of this measure (Jauch et al., 2013).

STK-2: discharged on antithrombotic therapy such as aspirin and STK-5: prescribing antithrombotic therapy in the hospital by the end of hospital day 2 . These are essential to stroke care because antithrombotic therapy assists in decreasing the risk of stroke recurrence, as well as morbidity and mortality (Jauch et al., 2013). Current data by the AHA demonstrates a small significant decline in mortality and unfavorable patient outcomes, including stroke recurrence, when aspirin is taken after a stroke (Jauch).

STK-3: anticoagulation therapy such as Coumadin for stroke patients with atrial fibrillation/flutter. According to the 2014 AHA guideline for management of patients with atrial fibrillation, patients must be prescribed anticoagulants (Jauch et al., 2014). Atrial fibrillation significantly increases the risk of ischemic stroke. Oral anticoagulation reduces the risk of stroke by $64 \%$ in patients with atrial fibrillation/aflutter. Atrial fibrillation is a common irregular heartbeat that over 
2.7 million Americans have. People may not know it puts them at a higher risk for stroke (Joundi, Cipriano, Sposato, Saposnick, 2014).

STK-4: thrombolytic therapy. Use of thrombolytic therapy minimizes disability by $30 \%$ (Jauch et al., 2013). Every 100 patients who delay thrombolytic therapy by 10 minutes are at an increased risk for disability (Saver et al., 2010). According to the AHA, $40 \%$ of patients treated with tPA were discharged home and $38.7 \%$ ambulated independently at discharge (Sheth et al., 2015). The required administration time is within 60 minutes of arriving to emergency room or time that code stroke is initiated on the inpatient floor. Tissue- Plasminogen Activator (tPA) is the thrombolytic given to stroke patients.

STK-6: discharged on statin medication. Statins, cholesterol-lowering medications, slow the progression of atherosclerosis, stabilizes plaque, reduces risk of rupture and risk of arterial thrombus therefore improving prognosis. Statins reduce the risk of stroke by $30 \%$. All patients with established cerebral disease should be prescribed a statin in conjunction with lifestyle changes (Jauch et al., 2013).

STK-8: stroke education. More than $90 \%$ of patients who seek treatment for stroke symptoms are not eligible for fibrinolytic therapy due to not seeking treatment within the desired time frame. This delay is due to lack of lack of awareness of stroke symptoms. It is imperative to educate all individuals to seek treatment immediately if symptoms reappear (Jauch et al., 2013). Patients should also be educated regarding importance of follow-up care and medication 
compliance. Data demonstrates that the public's knowledge of onset of stroke symptoms remains poor. Less than half of patients who call EMS recognize their symptoms for possibly being caused by a stroke. In order to ensure the likelihood of decreased disability from stroke the first step is early recognition (Jauch et al., 2013).

STK-10: assessed for rehabilitation. Stroke patients are at risk for serious complications caused by immobility. Early ambulation and physical therapy decreases stroke disability (Goldszmidt \& Caplan, 2010). A Very Early Rehabilitation Trial for Stroke (AVERT) was a large randomized controlled trial that mobilized patients within the first 24 hours after stroke. The pilot program demonstrated that early mobilization of stroke patients resulted in fewer complications such as DVT, Pneumonia and pressure ulcers. Early mobilization also decreases the risk for contractures and orthopedic complications (Jauch et al. 2013).

\section{Get with the Guidelines - Stroke}

Separate from the TJC core measures, the AHA program, Get with The Guidelines $(G W T G)$ is a hospital program for improving stroke care by promoting consistent adherence to the most current scientific treatment guidelines. Get with The Guidelines is not affiliated with TJC. Numerous published studies demonstrate the AHA program's success in achieving measurable patient outcome improvements Get with The Guidelines is an American Heart Association program. Get with The Guidelines was initiated in 2003 and since then over 1,500 hospitals have entered more than two million patient 
records into the Get With The Guidelines-Stroke database (AHA, 2016). From 2003 to 2007 a study of 322,847 hospitalized stroke patients in 790 US academic and community hospitals voluntarily participated in GWTG-stroke program showed significant improvement in stroke care. Improvements included tPA administration increased from $42.9 \%$ to $72.84 \%$, prescribed antithrombotic within 48 hours increased $91.46 \%$ to 97.04\%. DVT prophylaxis increased from $73.89 \%$ to $89.54 \%$, and anti-coagulation treatment for atrial fibrillation increased also (Jauch, et al., 2013).

The goal of the AHA program is to ensure positive patient outcomes when evidence-based medicine is practiced. Silver, Gold, Silver Plus and Gold Plus Awardwinning Get With The Guidelines-Stroke hospitals are honored at national recognition events during the International Stroke Conference and listed by name in advertisements that appear annually in the journal Stroke and in the "Best Hospitals" issue of U.S. News \& World Report. All award-winning hospitals are provided customizable marketing materials they can use to announce their achievements locally (AHA, 2015).

Next, the theoretical framework will be presented. 


\section{Theoretical Frameworks}

The AACN Synergy model for patient care and the Logic model were used to guide this program development, quality improvement project.

\section{The American Association of Critical-Care Nurses (AACN) Synergy Model for Patient Care}

The AACN Synergy Model for Patient Care (Curley, 2007) is a conceptual model that when nurse competencies are in synergy with patient characteristics patients have optimal outcomes. The eight patient characteristics are resiliency, vulnerability, stability, complexity, resource availability, participation in care, participation in decision-making and predictability. Each characteristic exists on a continuum from low (level 1) to high (level 5) (Curley, 2007).

The Synergy model describes eight nurse competencies that include clinical judgment, advocacy/moral agency, caring practices, collaboration, system thinking, responses to diversity, clinical inquiry and facilitator of learning. The nurse competencies are influenced by three spheres of practice: patient; nurse; and system. The model is based on five assumptions: (1) patients are biological, social, and spiritual entities who present at the particular development stage. The whole body including the mind, body and spirit must be considered; (2) the patient, family and community all contribute to providing a context for the nurse-patient relationship; (3) patients can be described by a number of characteristic; all characteristics are connected and contribute to one another, characteristic cannot be looked at in isolation; (4) nurses can be described on a number of dimension and (5) a goal of nursing care is to restore a patient to optimal level of wellness or to move a patient toward a peaceful death. A core concept of the Synergy 
Model is that the needs of patients and families influence the nurse competencies. The goal of clinical judgment is to have the nurse be able to see the entire clinical situation and make decisions based on the entire clinical picture.

The Synergy model was used to develop a stroke education orientation program for nurses on a designated stroke unit. This model aligns with the goal of this program to assure nurse competencies meet stroke patient care needs. Evidence demonstrates that nurse education is not only mandatory but has proven, positive outcomes for patients cared for in a PSC.

\section{The Logic Model}

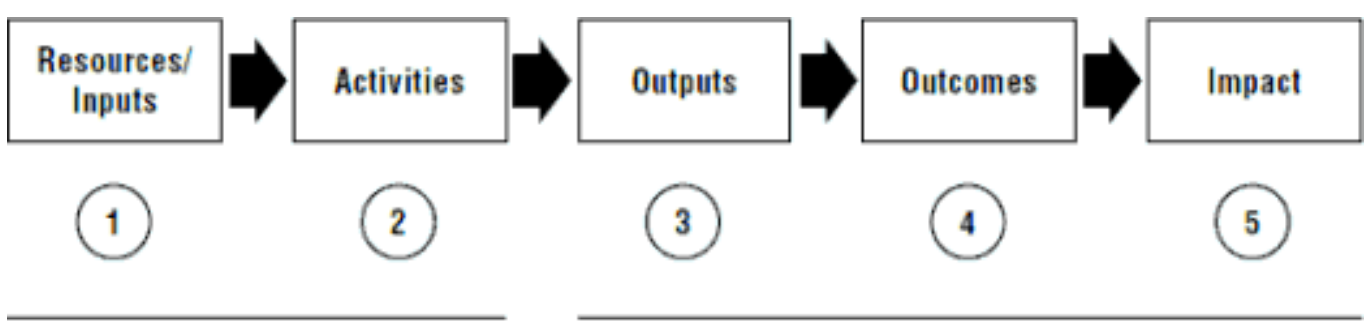

Your Planned Work

Your Intended Results

Figure 1. The Basic Logic Model.

The Logic Model (W.K.Kellogg Foundation, 2004) is an evaluation tool that assists with the development of programs. The Logic Model describes the sequence of events that has to take place in order to achieve desired outcomes. The model uses pictures and words and acts like a road map to follow a certain path to outcome. The model is clear, concise and provides an organized approach to program development and assists in illustrating and organizing the program. 
The Logic Model consists of a series of five steps. The first step identifies the resources/inputs that are available and needed to build the program. Resources include human, financial, organized and community-based resources. All these resources may limit or enhance the program. The second step is activities. Activities refer to what the program designer is going to do in order to accomplish the program. Activities include tools, events, technology and actions that are essential for the program implementation. Output is the third step and represents the intended result of the program. When this step is complete the developer will hopefully be able to deliver the product or service that was intended. The fourth step is outcomes, used to determine the participants have benefited from the program. This step includes assessing the behavioral outcomes and knowledge of the participants and sustainability of the program. The fifth and final step is impact. Impact is used to describe what changes might be expected to occur in the facility or organization.

Following the steps of the model keeps the program organized and on task. The model has been proven to provide a successful pathway to program development (Logic Model Development Guide, 2004) and was used to develop a Stroke Education Orientation Program for nurses on a designated stroke unit. 


\section{Methods}

\section{Purpose}

The purpose of this program development, quality improvement project was to develop a computer-based, stroke education orientation program for nurses on a designated stroke unit.

\section{Design}

The design of this quality improvement project was program development.

\section{Site and Sample}

When implemented, the computer-based program will be assigned to all new and current registered nurses, hired to work on the designated stroke unit at a primary stroke center, in a 250 bed, teaching, urban community hospital.

\section{Procedures}

Permission from the Center for Professional Practice and Innovation, the Department of Nursing and from the Stroke Program Manager at The Miriam Hospital was obtained.

\section{Implementation Plan Using the Logic Model}

The stroke education program was developed using the W.K. Kellogg Foundation's Logic Mode, as illustrated in Table 1. 
Table 1.

Implementing the Logic Model

\begin{tabular}{|c|c|c|c|c|}
\hline Resources/Inputs & Activities & Outputs & Outcomes & Impact \\
\hline $\begin{array}{l}\text { TJC Core } \\
\text { Measures for } \\
\text { Primary Stroke } \\
\text { Center } \\
\text { Certification } \\
\text { AHA/ASA 'Get } \\
\text { with the } \\
\text { Guidelines' best } \\
\text { practices } \\
\text { Stroke } \\
\text { Coordinator, } \\
\text { content expert } \\
\text { Nurse manager } \\
\text { of the new } \\
\text { inpatient stroke } \\
\text { unit } \\
\text { Current stroke } \\
\text { education } \\
\text { programs at } \\
\text { hospital } \\
\text { Time constraints } \\
\text { Assess current } \\
\text { resources } \\
\text { Program content }\end{array}$ & $\begin{array}{l}\text { Each core } \\
\text { measure will } \\
\text { be described } \\
\text { with evidence } \\
\text { based } \\
\text { rationale } \\
\text { GWTG will } \\
\text { be explained } \\
\text { Assess } \\
\text { current } \\
\text { available } \\
\text { stroke } \\
\text { education } \\
\text { resources } \\
\text { Review of } \\
\text { TMH current } \\
\text { policy, } \\
\text { procedures } \\
\text { and protocols } \\
\text { Program will } \\
\text { focus on TJC } \\
\text { core measures } \\
\text { with a brief } \\
\text { overview of } \\
\text { GWTG. } \\
\text { Program will } \\
\text { consist of } 4 \\
\text { (1) online } \\
\text { modules with } \\
\text { questions } \\
\text { built into the } \\
\text { modules to } \\
\text { assess } \\
\text { learning }\end{array}$ & $\begin{array}{l}\text { Meetings will } \\
\text { take place } \\
\text { throughout the } \\
\text { development } \\
\text { with NM and } \\
\text { Stroke } \\
\text { program } \\
\text { manager to } \\
\text { make sure } \\
\text { goals of } \\
\text { program and } \\
\text { management } \\
\text { align } \\
\text { 100\% of all } \\
\text { new RNs will } \\
\text { complete the } \\
\text { education by } \\
\text { the end of } \\
\text { their } 3 \text {-month } \\
\text { orientation, } \\
\text { current nurses } \\
\text { will have } 30 \\
\text { days to } \\
\text { complete }\end{array}$ & $\begin{array}{l}\text { The education } \\
\text { program will } \\
\text { focus on } \\
\text { education } \\
\text { topics and } \\
\text { materials that } \\
\text { essential to } \\
\text { acute stroke } \\
\text { care to ensure } \\
\text { that nurses } \\
\text { have the } \\
\text { expertise to } \\
\text { care for these } \\
\text { patients. } \\
\text { Long-term } \\
\text { outcome: } \\
\text { TMH will } \\
\text { receive the } \\
\text { AHA GWTG } \\
\text { award } \\
\text { TMH will } \\
\text { maintain } \\
\text { recognition as } \\
\text { a PSC }\end{array}$ & $\begin{array}{l}\text { Nurses on the } \\
\text { designated } \\
\text { stroke floor will } \\
\text { have the } \\
\text { knowledge to } \\
\text { provide care } \\
\text { according to } \\
\text { evidence based } \\
\text { practice } \\
\text { national } \\
\text { guidelines and } \\
\text { be able to be } \\
\text { recognized as } \\
\text { GWTG award } \\
\text { recipient and } \\
\text { have impact on } \\
\text { the hospital } \\
\text { maintaining } \\
\text { their stroke } \\
\text { certification } \\
\text { All stroke } \\
\text { patients will } \\
\text { receive current, } \\
\text { accurate and } \\
\text { high quality } \\
\text { stroke care } \\
\text { Improved } \\
\text { communication } \\
\text { among } \\
\text { interdisciplinary } \\
\text { team }\end{array}$ \\
\hline
\end{tabular}


Needs Assessment. A designated stroke unit with knowledgeable staff is a requirement of certification. Initial orientation and annual education of nurses is required. Education available at this time is given in real time and is task focused. A comprehensive education program is not available at this time. An up-to-date stroke RN orientation program is identified as a goal by the stroke unit management team. Currently the hospital is not recognized as an American Heart Association Get With The Guidelines - Stroke award recipient.

Resources and Inputs. The resources used for this program were the TJC core measures for stroke care, AHA/ASA and evidence-based research. The program provides an overview of the GWTG. The Stroke Program Manager at The Miriam Hospital evaluated the program content as it was developed and served as a resource as content experts.

Activities. The activities included assessing resources that currently exist. There was not an up-to-date, comprehensive, structured stroke orientation program in place for nurses on the newly designated stroke unit. It was decided that a computer-based program would be most beneficial to allow the nurses to complete the education online, which gives them the flexibility to learn at their own pace. This program was developed using TJC Core measures as a guideline with an overview of the American Heart Association Get with the Guidelines. Each core measure is described at length including rationale and evidence that supports each core measure. Nursing specific interventions are described along with the evidence and current best practice standards as put forth by the AHA/ASA. The objectives for each module were based upon the required information that nurses working on a stroke unit needs in order to care for stroke patients. 
The modules are divided into four modules addressing stroke physiology, types of stroke, explanation of TJC core measures and protocols and policies specific for The Miriam Hospital.

Meetings were held with the Stroke Program Manager throughout the development of the program to ensure that the program aligned with management goals. The computer-based program contains four online modules with content objectives, questions and case studies embedded within the modules to assess knowledge for each module. The Stroke Program Manager validated the module content.

Outputs. The scope of this quality improvement project included development of the computer-based learning modules only. The stroke coordinator will complete implementation of the four modules. The stated intent of management is that $100 \%$ of all RNs on the designated stroke unit will complete the education. Nurses currently employed on the designated stroke unit will have 30 days to complete the training. Newly hired RNs will complete the training by the end of their 90-day orientation probationary period.

Outcomes. The education program focuses on competencies and resources essential for high quality acute stroke care and designed to ensure nurses have the expertise to care for these patients. The long-term desired outcome is that The Miriam Hospital will receive the AHA GWTG award and maintain recognition as a PSC.

Consistent with the 8 nursing characteristics of the Synergy model, clinical inquiry is ongoing and the education incorporates the AHA guidelines for stroke patients. Caring practices engages the patient and family in care and is attentive to the usual and 
needs of the patient and family. Stroke is devastating and education regarding care and prevention is essential for optimal patient outcomes. Response to diversity is included in the education so that nurses can individualize stroke patient care according to a patient's risk factors. Advocacy is exercised in the care of stroke patients because nurses orchestrate and coordinate care within the interdisciplinary teams to meet individual patient care needs. The nurse also is the facilitator of learning. Patient education is key and recognizing the level of patient learning and style is vital to delivering patientcentered teaching. Collaboration is an essential nurse competency. Nurses are at the center of patient care, facilitating the contributions of all team members. Systems thinking is role modeled by implementing best practices as they are developed by The Joint Commission and The American Heart Association. Knowledge of the certification requirements and how they affect an institution is a vital aspect of the education program.

Impact. Nurses on the designated stroke unit will have the knowledge to provide care to meet evidence-based practice, national guidelines and the ability to meet the standards to be recognized as GWTG award recipient and have positive impact on PSC certification. PSC and GWTG designations ensure that all stroke patients will receive upto-date, accurate and high-quality stroke care and improved communication among interdisciplinary team members. The stroke education orientation program for nurses on a designated stroke unit: program development, the Stroke Program Manager on the designated inpatient stroke unit will implement quality improvement project. The Stroke Program Manager will evaluate the program content, nurse evaluations, tests and stroke program outcomes. 


\section{Results}

The educational program is divided into four, online computer-based modules with questions embedded in the modules. All four modules are found in the appendices

A., B., C., and D. It is estimated that each module will take approximately 60 minutes to complete.

Table 2.

Objectives of the Stroke Modules

\begin{tabular}{|c|c|}
\hline Module & Objectives \\
\hline Module One & $\begin{array}{l}\text { Recognize signs, symptoms and risk } \\
\text { factors of a stroke } \\
\text { List four actions to take during a code } \\
\text { stroke according to The Miriam Hospital } \\
\text { Protocol } \\
\text { State components of tPA administration } \\
\text { protocol } \\
\text { Describe components of the National } \\
\text { Institutes Health Stroke Scale }\end{array}$ \\
\hline Module Two & $\begin{array}{l}\text { Identify and recognize possible stroke } \\
\text { complications } \\
\text { State differences between primary Stroke } \\
\text { Center (PSC and comprehensive Stroke } \\
\text { center } \\
\text { Identify The Joint Commission (TJC) } \\
\text { stroke core measures and how they relate } \\
\text { to patient care. }\end{array}$ \\
\hline Module Three & $\begin{array}{l}\text { Define American Heart Association: Get } \\
\text { with the Guidelines (GWTG) }\end{array}$ \\
\hline
\end{tabular}




\begin{tabular}{|l|l|}
\hline Module Four & $\begin{array}{l}\text { Describe appropriate interventions to do } \\
\text { during a code stroke. }\end{array}$ \\
\hline & $\begin{array}{l}\text { Describe process of obtaining Nurse } \\
\text { certification }\end{array}$ \\
$\begin{array}{l}\text { Identify resources available through the } \\
\text { Lifespan Intranet }\end{array}$ \\
$\begin{array}{l}\text { Describe how certification aligns with } \\
\text { Magnet Hospital Designation. }\end{array}$ \\
\hline
\end{tabular}




\section{Summary and Conclusions}

Stroke is a devastating disease. Stroke is the $4^{\text {th }}$ leading cause of death in the U.S., and can result in permanent disability and complications. $80 \%$ of strokes are ischemic (Curley, 2007). Permanent disabilities secondary to ischemic stroke include dysarthria, aphasia, facial weakness and extremity weakness. Use of thrombolytic therapy minimizes disability by $30 \%$ (Jauch et al., 2013). In order to achieve optimal outcomes thrombolytic therapy must be administered within 60 minutes of symptom onset. Early recognition of stroke and timely intervention is vital to reduce these long-term disabilities. In clustered, controlled clinical trials outcomes in PSC hospitals, demonstrated better patient outcomes compared to non-certified hospitals (Jauch et al., 2013). According to TJC a PSC must have qualified, educated, stroke care trained nurses working on a designated stroke floor. Requirements established by TJC include 8 CEUs per year, initial education and yearly education. It is important that nurses working at the PSC have stroke care education to be equipped to provide high quality, timely care to a complex patient population. The Synergy Model of developing nursing competencies to meet patient needs provided the framework for the project.

The purpose of this program development, quality improvement project was to create an orientation program for nurses on a designated stroke unit. The newly designated stroke unit did not have a comprehensive educational program for the nurses hired to work on the unit. PSC are required to provide education to the staff at time of orientation and annually. Computer- based modules were chosen to allow the nurses to complete the program at their own pace and when it is convenient for them. The computer-based modules were aligned with TJC national core measures and provide 
evidence based best practices that support each core measure. The development of this program followed the Logic Model framework. After a needs assessment was performed, it was determined along with the Stroke Program Manager that an educational program was necessary.

The Stroke Program Manager will implement the program with current nurses and all new nurses who work on the stroke unit. Completion of the modules will validate nurses' knowledge and meet TJC requirements. The content will be updated annually to ensure information is current and aligned with most up-to-date, evidence-based standards.

The purpose of this Quality Improvement project was the design and development of the computer-based learning modules. Implementation of the four computer-based learning modules and follow-up evaluation of program objectives and outcomes will be completed by the Stroke Program Manager. Limitations are that participants could not be assessed to evaluate satisfaction because the program was not implemented.

In conclusion, leadership support is essential in stroke education. As a PSC facility, promoting knowledge in practice and expert skill in patients suffering stroke should be a fundamental part of the education program. Nurses must have the essential resources needed to obtain stroke specific skills and knowledge. Essential resources include financial support and computer accessibility. Employing a computer-based module design allows staff individual access and availability to complete education. The expectation is that nurses working on the designated inpatient unit will complete the program during their scheduled work hours. Newly hired nurses will have time set aside to complete the mandatory education during orientation. 
Additional stroke care related resources for nurses will be identified and for staff. The fourth on-line module contains links to The American Heart Association, The Joint Commission, the Lifespan Stroke Center website and the Lifespan Nurse Reference Center. All sites provide education and are available to nurses to enhance their knowledge. Identifying evidence-based websites will give them the opportunity to enhance their knowledge on specific topics of interest. 


\section{Recommendations and Implications for Advanced Nursing Practice}

McKinley describes the Clinical Nurse Specialist (CNS) as the clinical expert on advanced knowledge and innovation within nursing science. The CNS is an expert coach, consultant and researcher. Developing an educational program based on current evidence-based, national best practices is well within the expertise of the Clinical Nurse Specialist (McKinley, 2007).

In the acute care setting the Advanced Practice Registered Nurse (APRN) is responsible to assess protocols, treatment plans and clinical pathways, to ensure provision of the most current, reliable, evidence-based, high quality care practices. Advance practice nurses are expert communicators and educators. These qualities are necessary to provide high quality, clear and relevant education for nurses providing stroke care. The APRN collaborates with members of the interdisciplinary team to improve patient outcomes. It is the unique role of the APRN/stroke program manager to assess educational needs and provide education to the clinical staff. The stroke program manager is responsible to ensure evidence-based practice, high quality care for patients and maintain compliance with widely established national guidelines.

According to the American Nurses Credentialing Center's Model for Magnet Designation, the component of New Knowledge, Innovations, and Improvements challenges healthcare organizations to provide exemplary care. APRNs are uniquely educated and positioned within organizations to develop strategies to improve patient outcomes (Daniels, Johnson, \& Mackovjak, 2011). 
Aligning with the ANCC's definition of Magnet this comprehensive stroke education orientation project will increase the level of competency of the nurses managing the care of stroke patients by increasing the quality of care, safety and patient satisfaction.

Implications for APRN practice is that the APRN will facilitate the education and serve as an expert resource in stroke care. The APRN continuously evaluates current best-practices and national standards.

After program implementation, it is the responsibility of the APRN to collect data on patient outcomes to measure program effectiveness in meeting objectives and outcomes. After initial evaluation, this program may be adapted for other departments to use throughout The Miriam Hospital and Lifespan system.

Patient satisfaction scores are another data point that may be used to measure outcomes. Yearly review of the program to ensure program aligns with current evidenced-based care will be the responsibility the Stroke Program Manager. It is the author's recommendation that the number of certified nurses, before and after education program completion be tracked by the Stroke Program Manager. Nurse post-program evaluations may be obtained and used to adjust the program according to the needs of the nurses and the facilities. Awarding Continuing Education Units is also a future implication for this program. 


\section{References}

American Heart Association. (2016). Retrieved May 18, 2016, from American Heart Association: http://brainhealth.strokeassociation.org/

California Acute Stroke Pilot Registry (CASPR) Investigators. (2005). Prioritizing interventions to improve rates of thrombolysis for ischemic stroke. Neurology, 64(4), 654-659.

Caplan, L. (2016). Etiology, classification, and epidemiology of stroke. UpToDate. Retrieved from https://remote.lifespan.org/contents/,Danalnfo=www.uptodate.com+etiologyclassification-and-epidemiology-of-stroke?

Curley, M. (2007). Synergy: The unique relationship between nurses and patients. Indianapolis: Sigma Theta Tau International

Daniels, C. S., Johnson, J. R., \& Mackovjak, J. (2011). Presenting a successful stroke education model to meet the Joint Commission's Disease-Specific Care Certification. Journal for Nurses in Professional Development, 27(2), 74-80.

Davis, C. \& Lockhart, L. (2016). Update: Stroke guidelines. Nursing Management, 2433.

Elkind, M. (2009). TIA and stroke: pathophysiology, management, and prevention...transient ischemic attack. American Health \& Drug Benefits, 2(S8), S8-14 1p. 
Eigsti, J., \& Henke, K. (2006). Anatomy and physiology of neurological compensatory mechanisms. Dimensions of Critical Care Nursing, 25(5), 197-202.

Feldman, L. (1951). A positive approach to management of cerebro-vascular accident. Geriatrics, 6 (4), 214-220.

Fonarow, G. C., Smith, E. E., Saver, J. L., Reeves, M. J., Bhatt, D. L., Grau-Sepulveda, M. V., .. \& Schwamm, L. H. (2011). Timeliness of tissue-type plasminogen activator therapy in acute ischemic strokeclinical perspective. Circulation, 123(7), $750-758$.

Goldszmidt, A. \& Caplan, L. (2010). Stoke Essentials. Sudbury, MA: Jones and Bartlett.

Goyal, M. S., Hoff, B. G., Williams, J., Khoury, N., Wiesehan, R., Heitsch, L., ... \& Lee, J. M. (2016). Streamlined hyperacute magnetic resonance imaging protocol identifies tissue-type plasminogen activator-eligible stroke patients when clinical impression is stroke mimic. Stroke, 47(4), 1012-1017.

Jauch, E. C., Saver, J. L., Adams, H. P., Bruno, A., Demaerschalk, B. M., Khatri, P., ... \& Summers, D. R. (2013). Guidelines for the early management of patients with acute ischemic stroke a guideline for healthcare professionals from the American Heart Association/American Stroke Association. Stroke, 44(3), 870-947.

Joundi, C. T., Wann, L. S., Alpert, J. S., Calkins, H., Cigarroa, J. E., Conti, J. B., ... \& Sacco, R. L. (2014). 2014 AHA/ACC/HRS guideline for the management of patients with atrial fibrillation: executive summary: A report of the American 
College of Cardiology/American Heart Association Task Force on Practice Guidelines and the Heart Rhythm Society. Journal of the American College of Cardiology, 64(21), 2246-2280.

Lindley, R. (2008). The Facts Stroke. Oxford, NY: Oxyford University Press.

McKinley, M. (Ed.). (2007). Acute and critical care clinical nurse specialists synergy for best practices (1st ed.). St Louis: Saunders Elsevier.

National Stroke Association. (2016). Retrieved September 12, 2016 from the National Stroke Association: http://www.stroke.org/

Saver, J. L., Smith, E. E., Fonarow, G. C., Reeves, M. J., Zhao, X., Olson, D. M., \& Schwamm, L. H. (2010). The "Golden Hour" and acute brain ischemia presenting features and lytic therapy in > 30000 patients arriving within 60 minutes of stroke onset. Stroke, 41(7), 1431-1439.

Schwamm, L., Fayad, P., Acker, J. E., Duncan, P., Fonarow, G. C., Girgus, M., ... \& Saver, J. L. (2010). Ttranslating evidence into practice: A decade of efforts by the American Heart Association/American Stroke Association to reduce death and disability due to stroke a presidential advisory from the American Heart Association/American Stroke Association. Stroke, 41(5), 1051-1065.

Sheth, K. N., Smith, E. E., Grau-Sepulveda, M. V., Kleindorfer, D., Fonarow, G. C., \& Schwamm, L. H. (2015). Drip and ship thrombolytic therapy for acute ischemic stroke use, temporal trends, and outcomes. Stroke, 46(3), 732-739. 
The Joint Commission (2016). Retrieved March, 12, 2016 from The Joint Commission: https://www.jointcommission.org/stroke/

Towfighi, A., \& Saver, J. L. (2011). Stroke declines from third to fourth leading cause of death in the United States historical perspective and challenges ahead. Stroke, 42(8), 2351-2355

W.K.Kellogg Foundation. (2004, January). Logic model development guide [Guideline model]. Retrieved from W.K.Kellogg Foundation website: http://www.wkkf.org 


\section{Appendix A}

\section{Computer-based Module I}

Objectives: Recognize signs, symptoms and risk factors of a stroke, identify and list four actions to take during a code stroke according to The Miriam Hospital Protocol, the time sensitive steps of a code stroke, state components of tPA administration protocol and state components of the National Institutes of Health Stroke Scale.

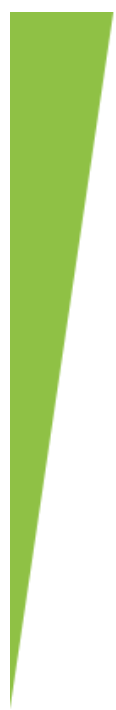

\section{Stroke Education} Module one

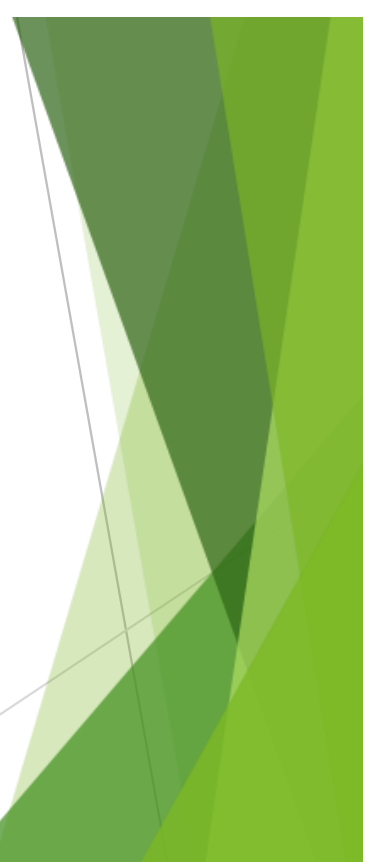




\section{OBJECTIVES}

- Recognize signs, symptoms and risk factors of a stroke

- List four actions to take during a code stroke according to The Miriam Hospital Protocol

- State components of tPA administration protocol

- Describe components of the National Institutes of Health Stroke Scale

\section{In The United States}

- Someone has a stroke every 40 seconds

- A person dies of a stroke every 4 minutes

- 1 in 20 deaths result from a stroke

$\checkmark 5^{\text {th }}$ leading cause of death

- 3.8 million women and 3 million men are living with a disability directly related from a stroke
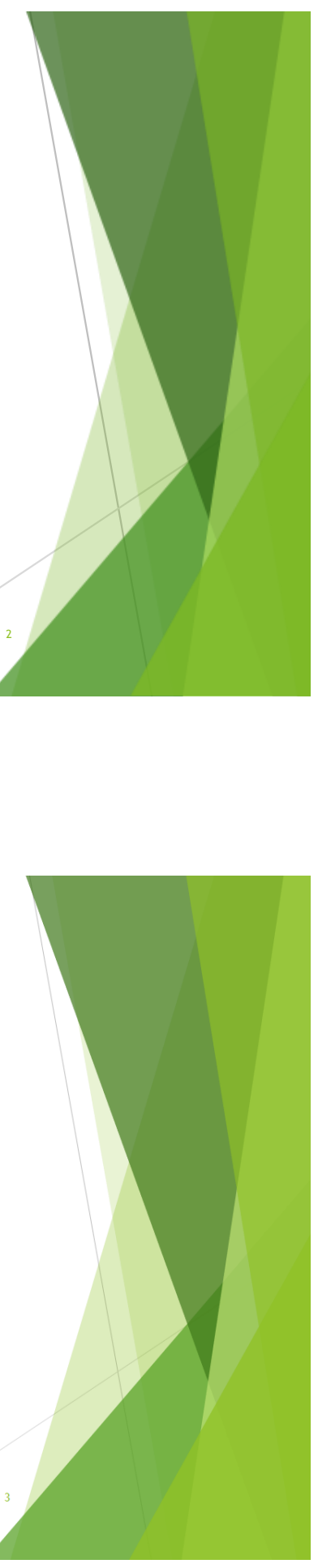


\section{Risk Factors}
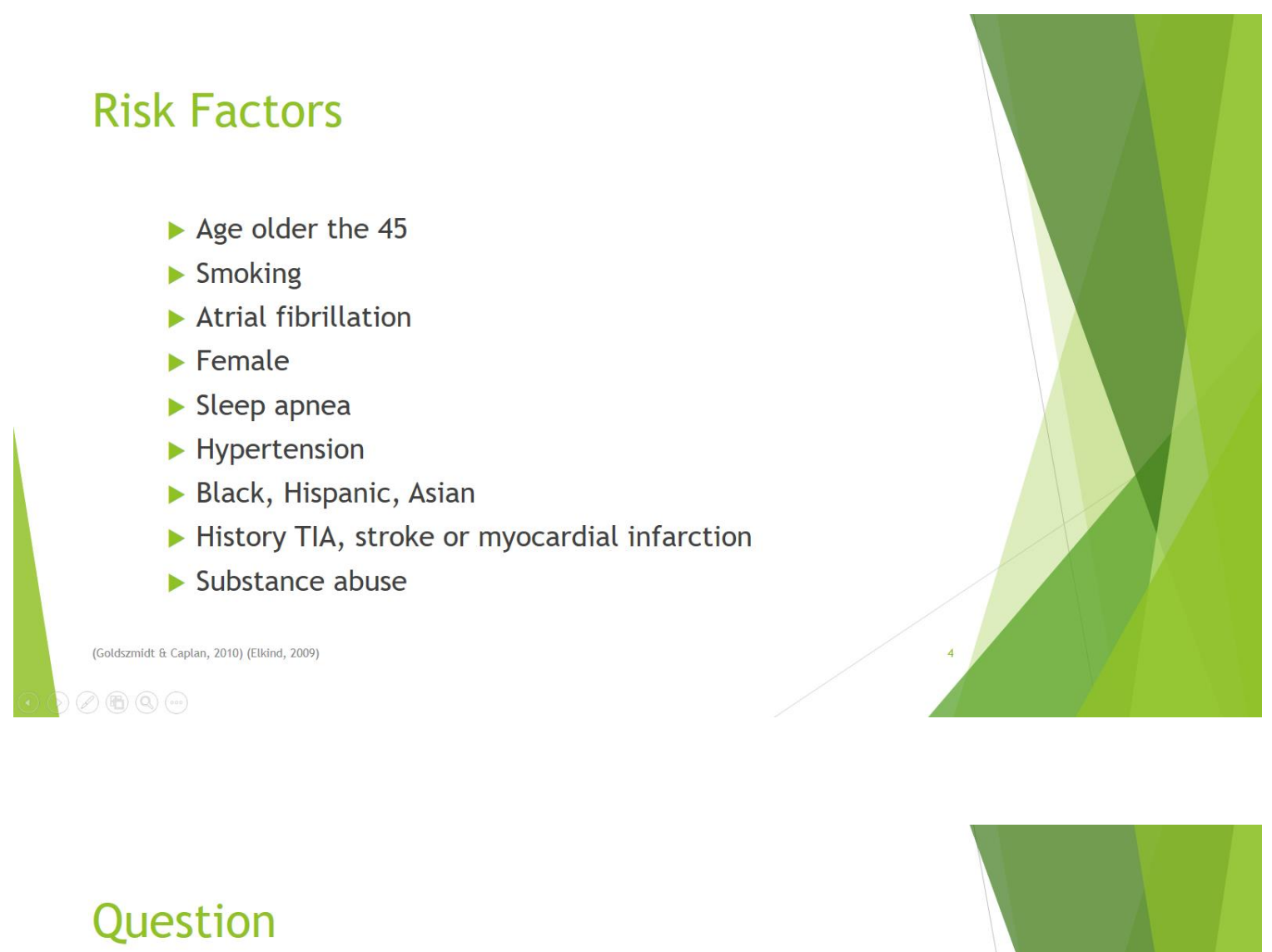

- What are two important types of stroke?
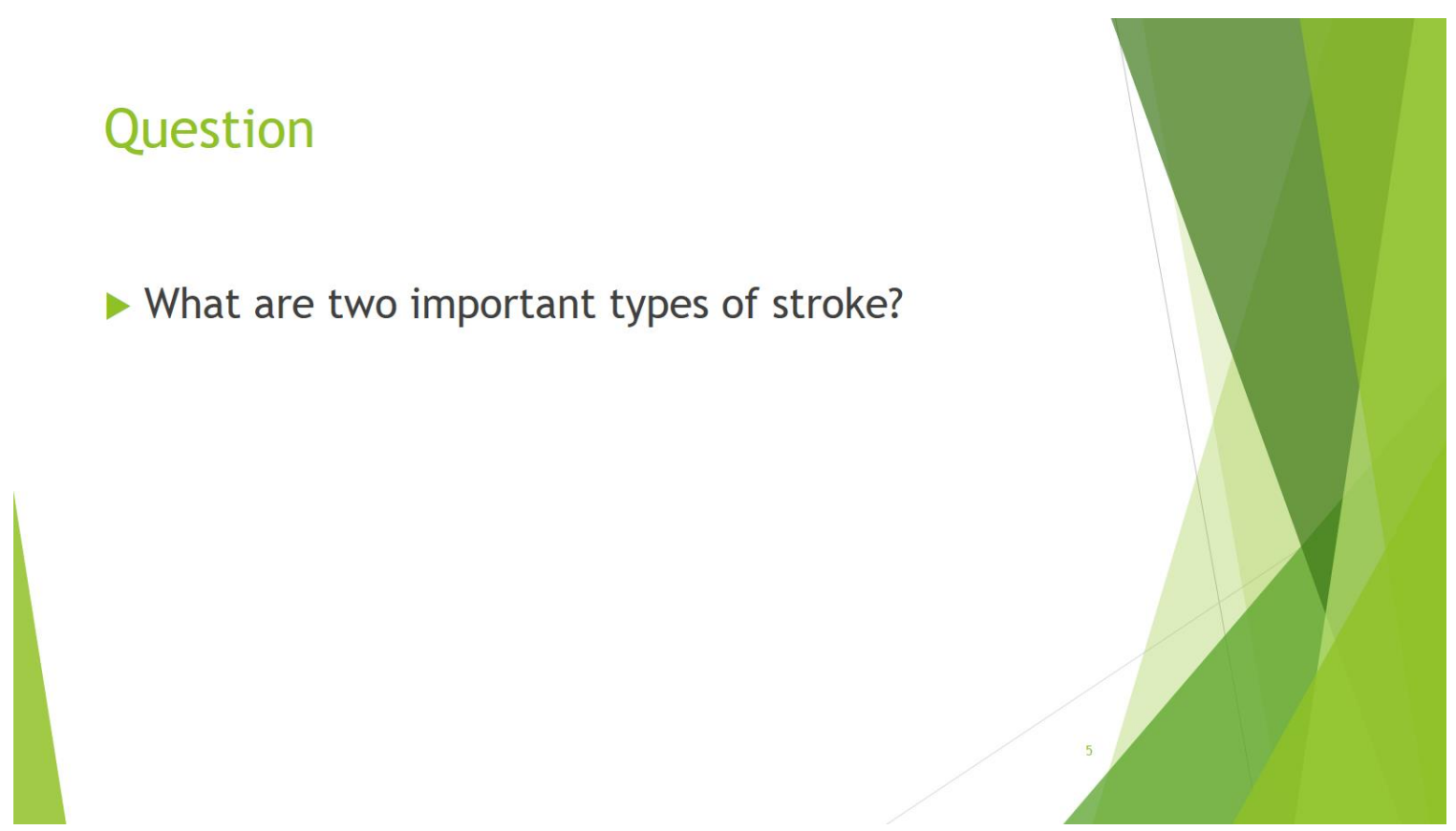


\section{Two Important Types}

- Hemorrhagic: occurs when areas of the cerebral arterial system become weaken secondary to long-term or acute episodes of increased pressure. The weakened thin area then evolves into an aneurysm and ruptures as pressure increases. The pressure then occludes blood flow to areas of the brain. Intracerebral hemorrhages are typically caused by rupture of vessels due to long-term atherosclerotic damage

- Atherosclerosis is plaque build up in the arteries that carry oxygen throughout the body. The plaque hardens over time and narrows the artery which then decreases the blood flow

\section{Two Important Types}

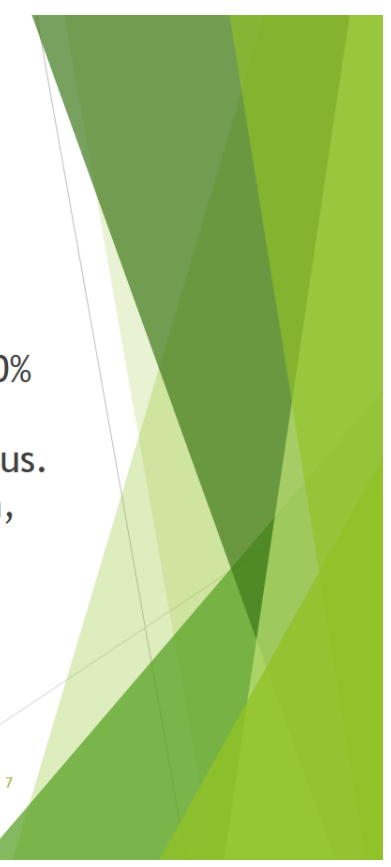

Ischemic stroke: the most common stroke and occurs in $80 \%$ of strokes, where the cerebral artery is occluded and deprives the brain of blood flow. This is caused by thrombus. The thrombus causes both glucose and oxygen deprivation, with subsequent brain cell death
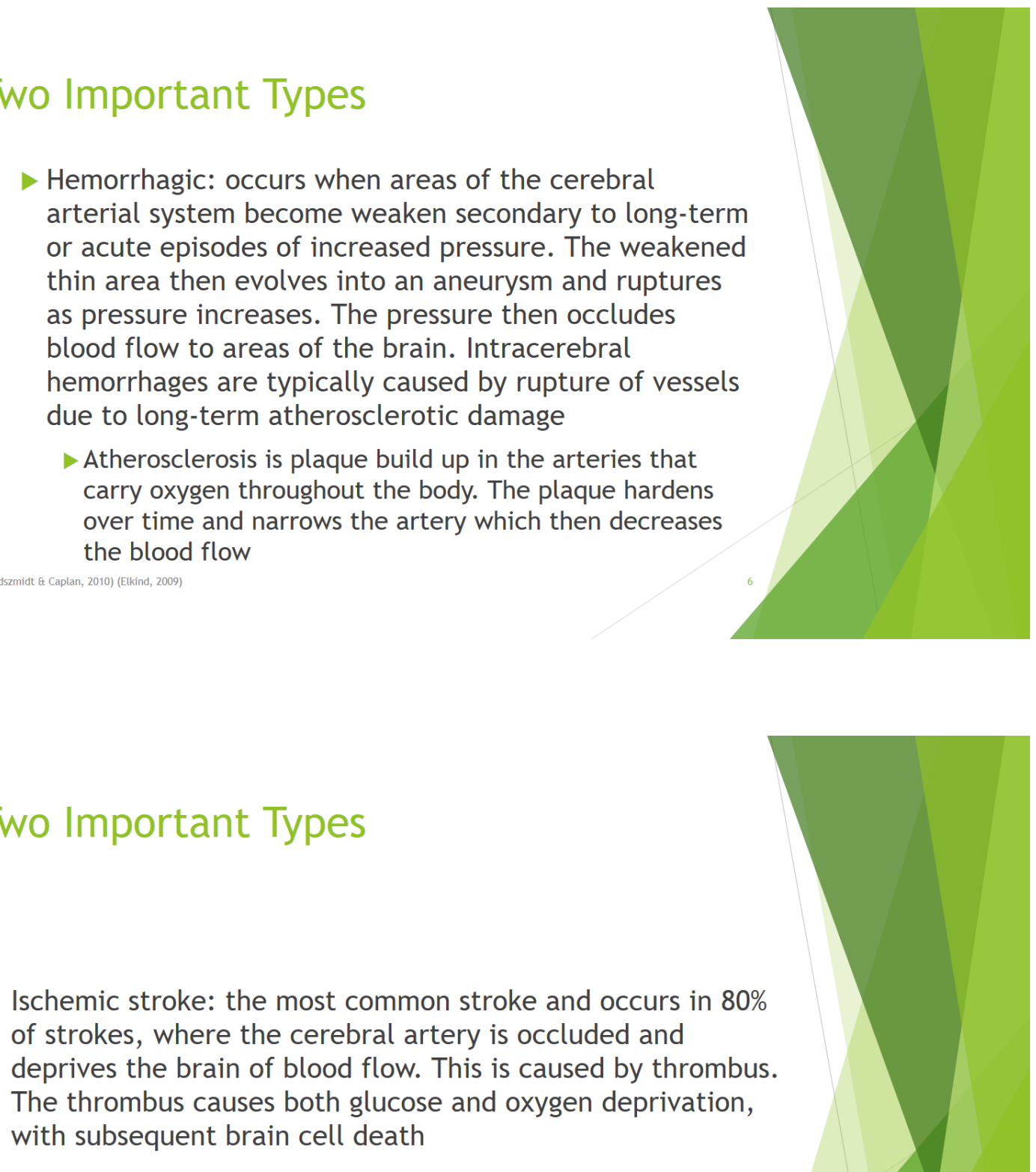


\section{Ischemic Stroke}

\section{Ischemic Stroke}

Occurs when oxygen-rich blood flow to the brain is restricted by a blood clot or other blockage.
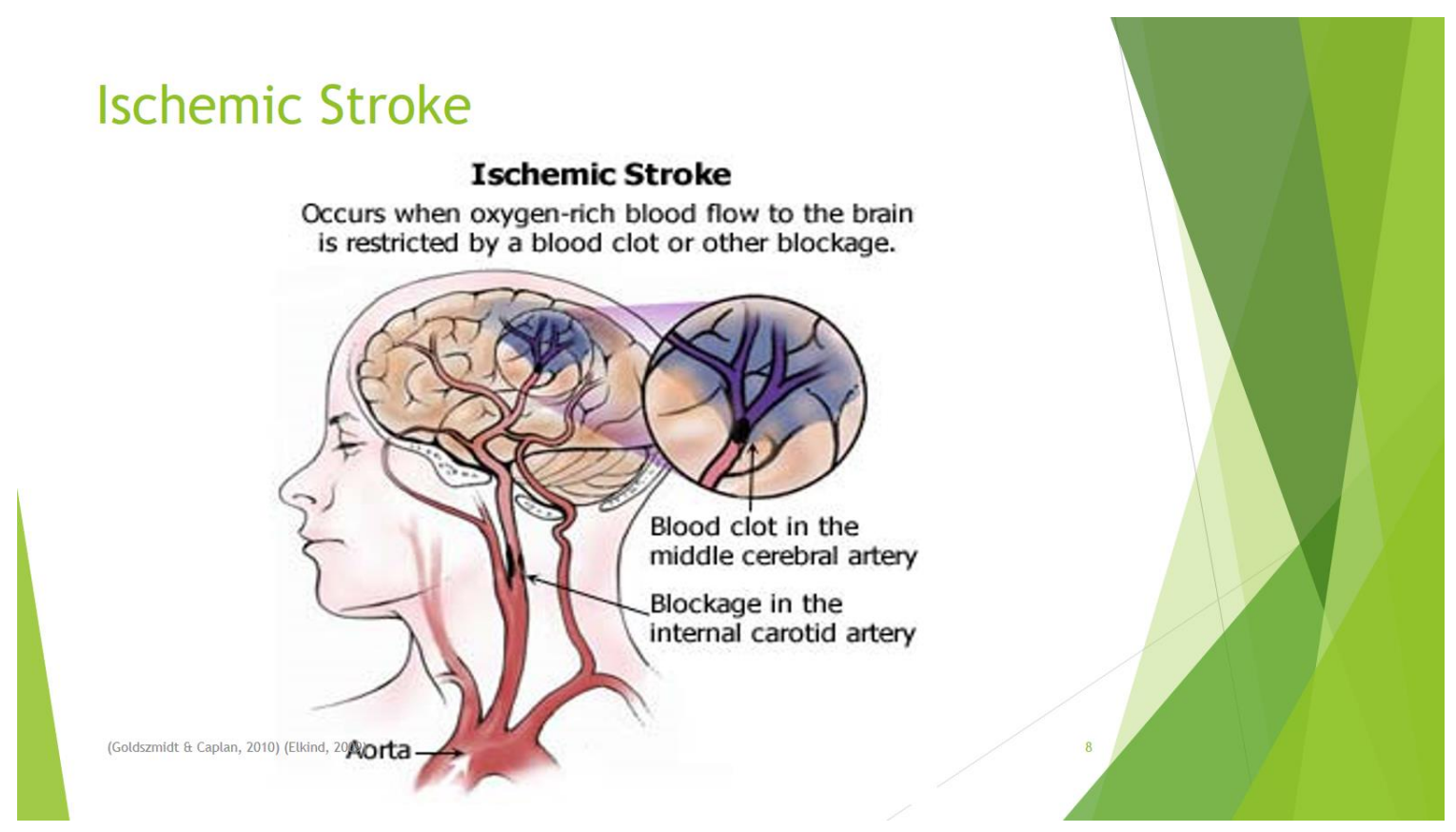

\section{Ischemic and Hemorrhagic}

- It is important to diagnosis what type of stroke the patient is having because type determines treatment

- Immediate treatment may minimize

$\checkmark$ long term effects

$\checkmark$ decrease chance of death

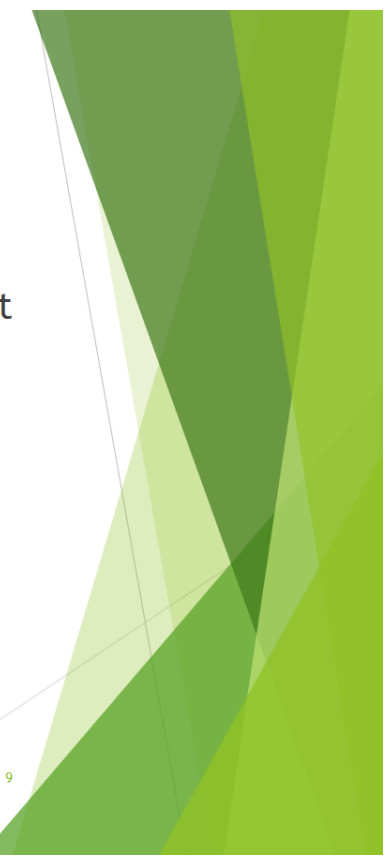


During hourly rounds you enter a patient's room and find your patient is having trouble speaking with right sided weakness

\section{What do you do?}
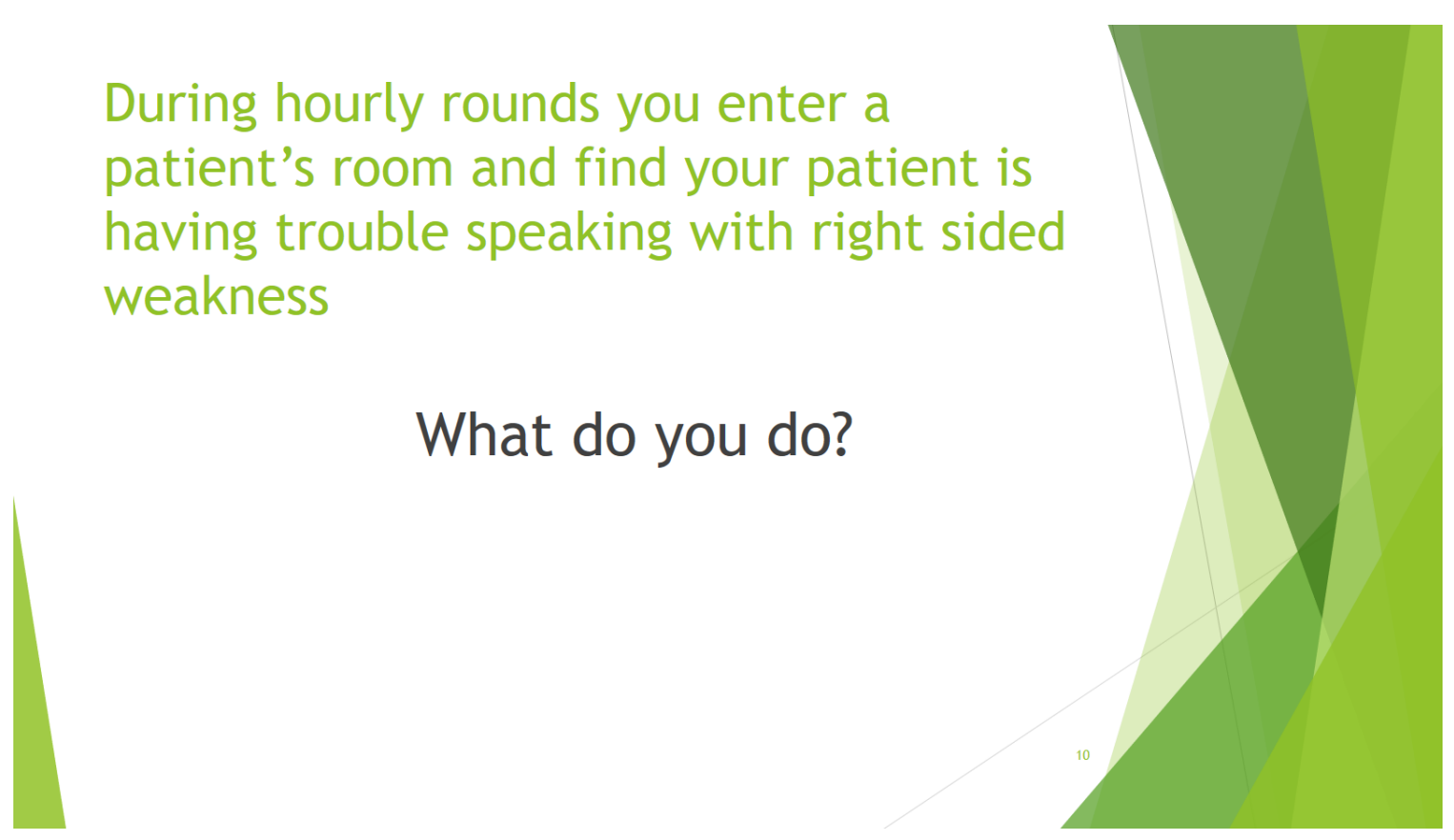

Think F.A.S.T.

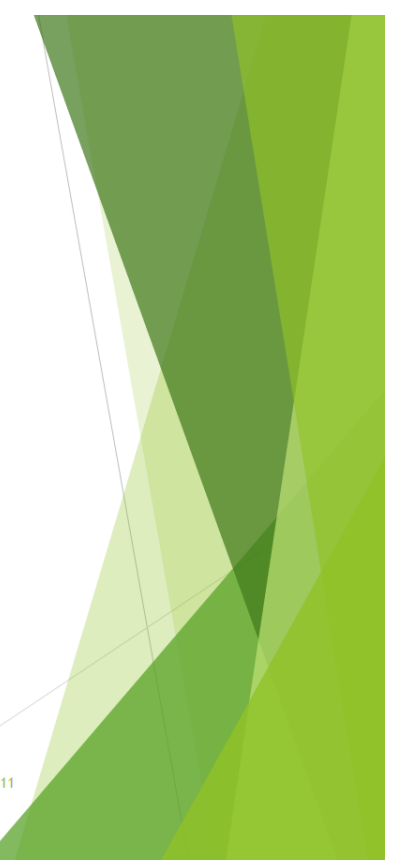

https: / / www.youtube.com/ watch?v=oyzwfsdd4AE

(AHA, 2016) 

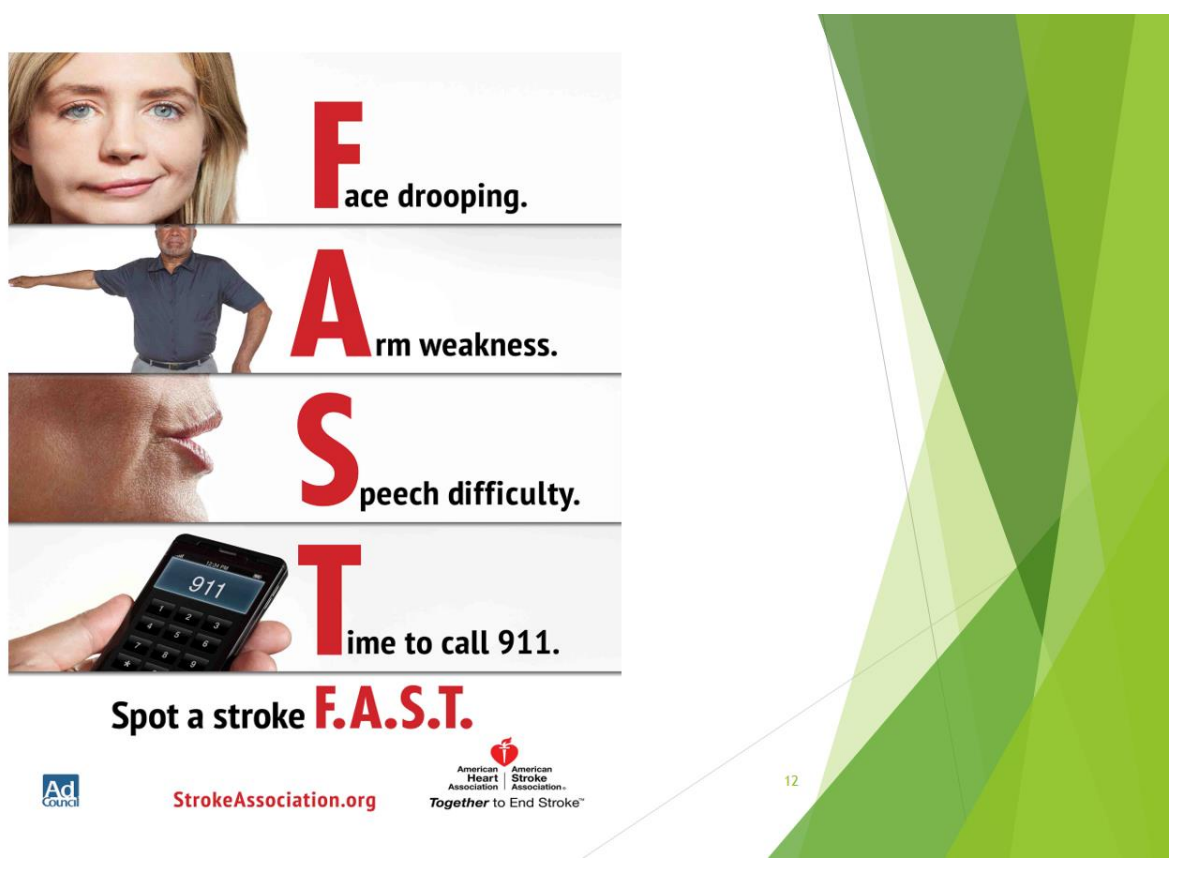

\section{Code Stroke}

- Call 3-5111 and ask operator to activate Rapid Response Team and code stroke

- Page the responsible MD/PA/NP - IMPORTANT: page LIP responsible for the patient, even if other LIPs respond to the RRT and code stroke page

- Perform Neuro and vital signs- document of RRT downtime form

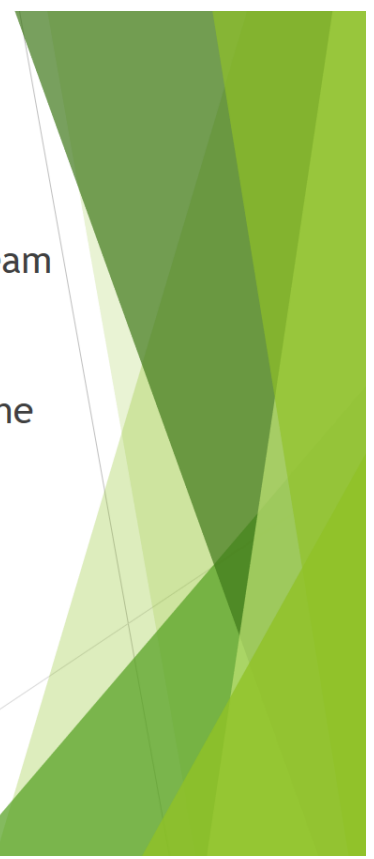




\section{Simultaneously}

\section{(but should not delay CT scan)}

- Attach portable cardiac monitor and prepare for transport to CT scan

- RN travels with patient to CT scan

- Obtain Blood Sugar, T\&S, Coags, EKG, VS, Neuro checks

- Oxygen for pulse ox $93 \%$ or less

- Obtained weight and Last Known Well (LKW) must be obtained for tPA (alteplase) administration

- NIHSS must be completed prior to tPA (alteplase) administration or within 48 hours after stroke

- Stroke Swallow Screen before any food, water, or medications

- If any of the above can't be done - document reason

\section{Time Sensitive Interventions.... .}

...the clock begins when code stroke is called or when the patient arrives in ED,

\section{within:}

- 10 minutes - must be seen by physician

- 15 minutes - must be seen by Stroke Team

- 25 minutes - CT scan completed

- 45 minutes - CT scan read

- 60 minutes- tissue plasminogen activator tPA, (alteplase) administration, if indicated (Door to needle, DTK
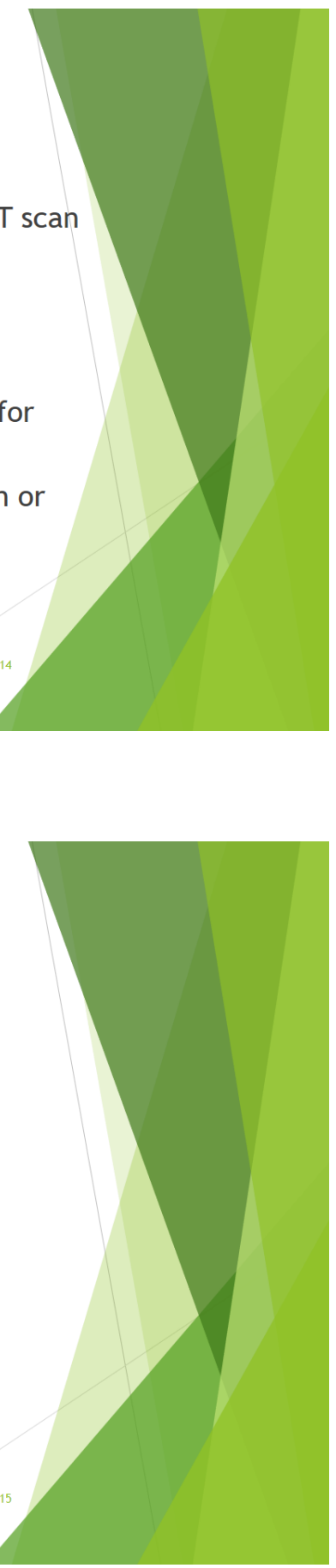


\section{Question}

- What type of stroke is defined as a sudden loss of function resulting from obstruction of blood flow due to clot or embolism?

\section{Answer \\ - Ischemic stroke}
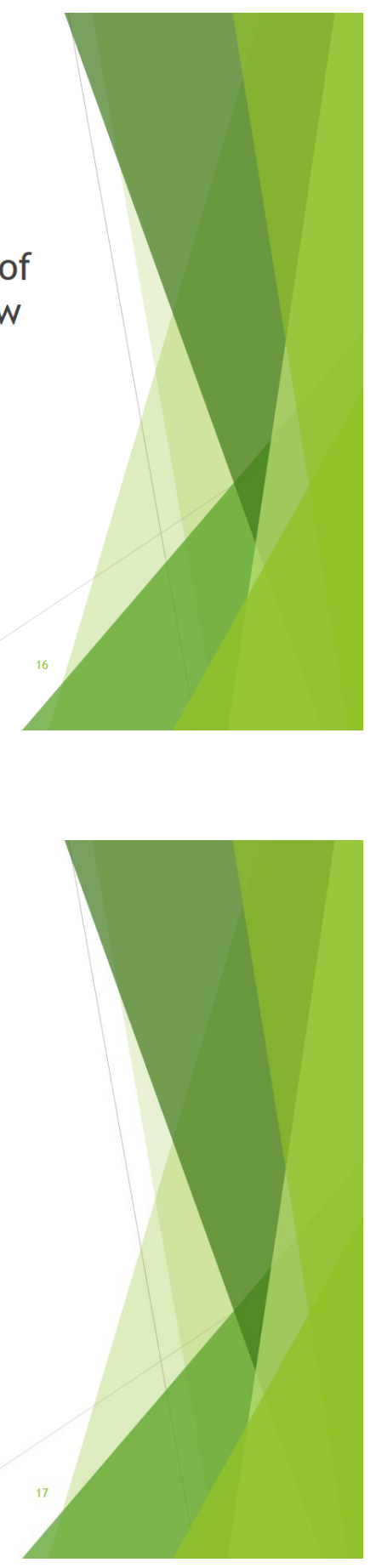


\section{CT Scan: Ischemic Stroke}

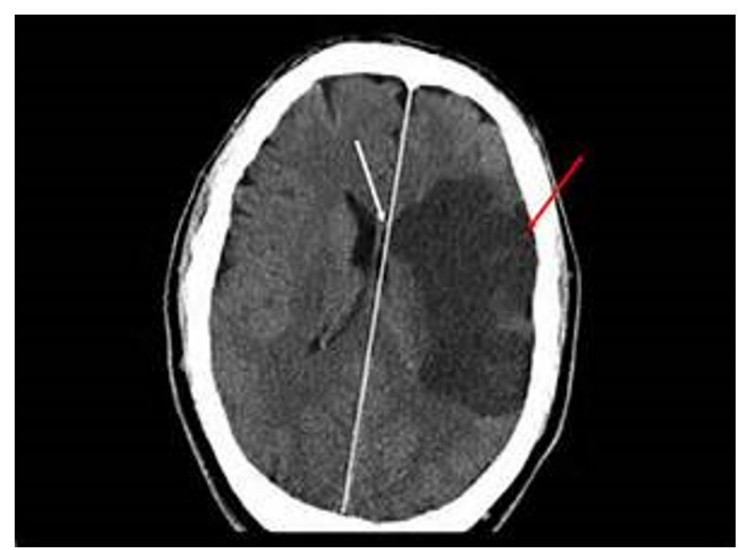

Dark area on the right represents the part of the brain that was affected by lack of blood supply

\section{TIME IS BRAIN}

Early intervention when stroke is suspected is vital to optimize neurologic outcomes
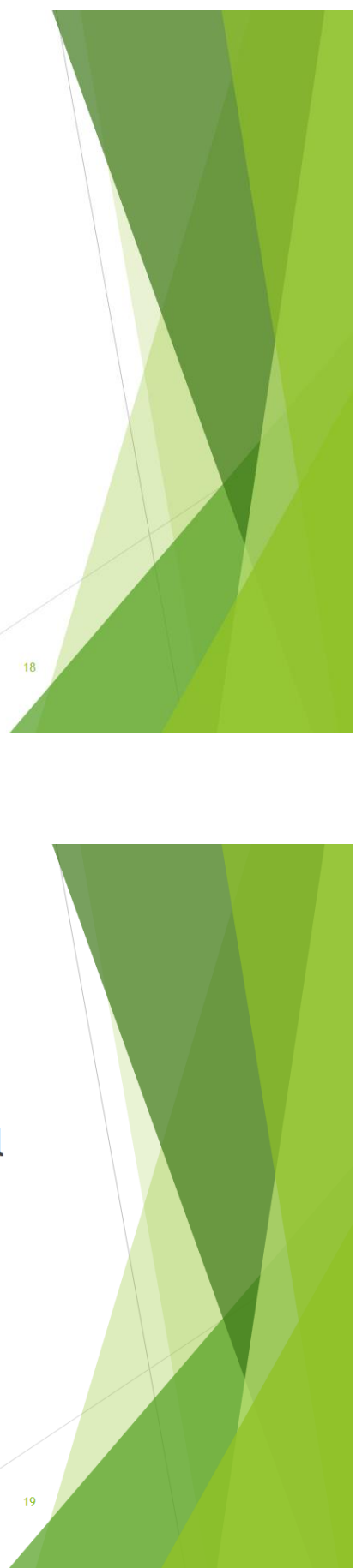


\section{Answer}

Call code stroke 3-5111

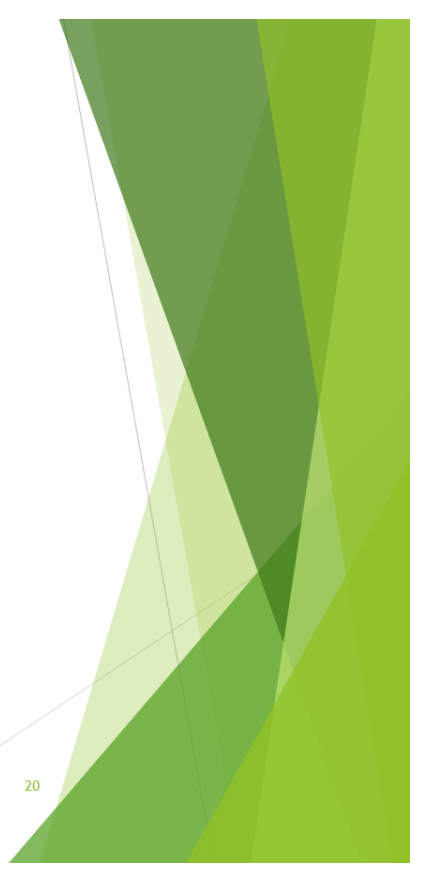

\section{Tissue Plasminogen Activator (tPA)}

- Treatment of choice for ischemic stroke. tPA is contraindicated in hemorrhagic stroke because it can expand the bleed and worsen the stroke

- tPA is the only FDA-approved drug for ischemic stroke

$\checkmark$ tPA ONLY given if Both:

- Systolic BP must be below 185 and,

Diastolic BP must be below 100

Notify LIP patient BP is out of range

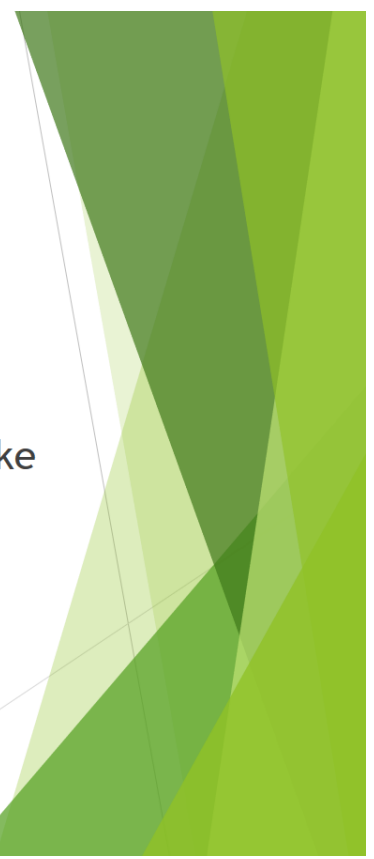




\section{Tissue Plasminogen Activator (tPA)}

- tPA is a fibrinolytic that stimulates the production of the enzyme plasmin, which digests fibrin strands and restores oxygen- rich blood flow to the brain

- Most effective in preventing permanent disability when given within 60 minutes of Last Known Well (LKW) but may be given up to 4.5 hour after LKW

- CAUTION: FDA reported in September 2015 that there have been 21 cases of wrong drug errors when orders have been written using the abbreviation "tPA" (alteplase). FDA recommends not using the abbreviation tPA. Miriam has replaced tPA with

$(\pi, 2015)$ Alteplase in all their policies

\section{Alteplase (tPA) Adminstration}

- tPA dose is calculated as $0.9 \mathrm{mg} / \mathrm{kg}$. $10 \%$ of the dose is given in a bolus over 1 minute with the remaining given over 60 minutes via IV pump

- Neurological status should be assessed every 15 minutes for 2 hours, every 30 minutes for 6 hours, then hourly for 16 hours

- Blood pressure should be maintained at less than $180 / 100 \mathrm{mmHg}$

- Patients who receive tPA are at risk for tPA associated hemorrhage and recurrent stroke due to hemorrhage. If neurological status declines, tPA should be stopped immediately and patient should have CT scan to rule out hemorrhagic conversion 


\section{Tissue Plasminogen Activator (tPA)}

- Ordering provider must discuss risks/benefits with patient/family

- LIP must document discussion in chart

- Ensure order is entered

- RN call pharmacy to ensure it is being delivered to bedside

- Post administration: full set of VS Q15 $\min \times 2 \mathrm{hr}, \mathrm{Q} 30 \mathrm{~min}$ x $6 \mathrm{hr}$, Q1 hour x16 hours

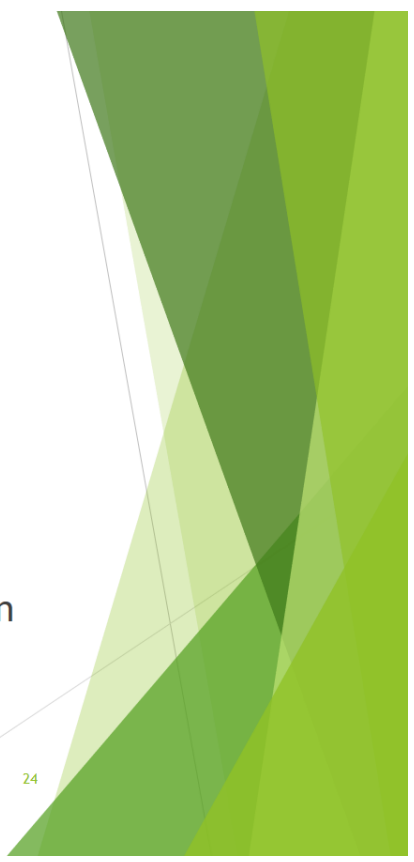

\section{National Institutes of Health Stroke Scale (NIHSS)}

- NIHSS is an assessment tool completed by clinicians who are certified by American Stroke Association, only

- Scale focuses on 15 specific areas to identify disabilities

- Must be completed prior to Alteplase (tPA) administration

$\checkmark$ If tPA (alteplase) is not administered due to contraindication as determined by provider and coordinating with neurologist, the reason for contraindication must be documented

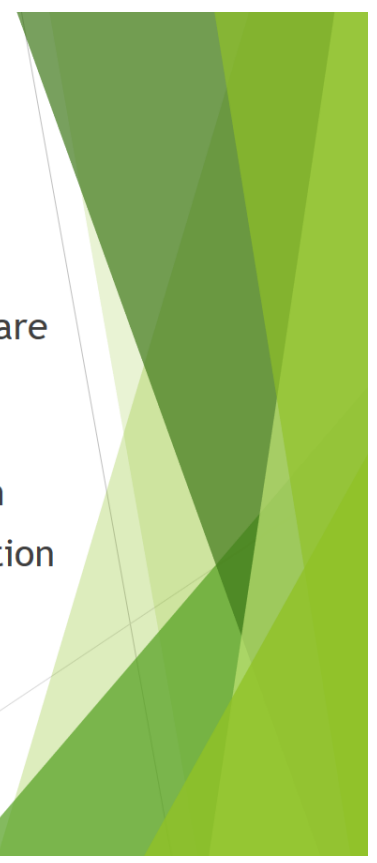




\section{National Institutes of Health Stroke Scale} (NIHSS)

- The score correlates with the size of infarction

- The score ranges from 0 to 42

- Score of 4 or less have a higher likelihood of functional independence

- Score of 22 or more may have severe disability

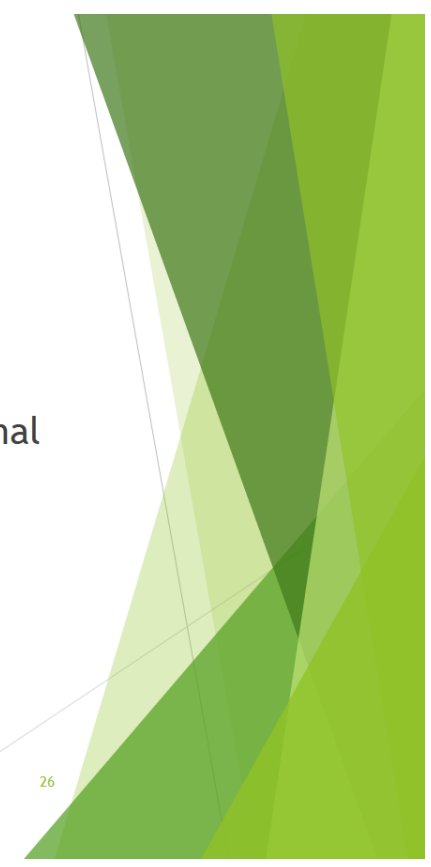

\section{National Institutes of Health Stroke Scale (NIHSS)}

- Clinicians that perform the NIHSS must be certified by the American Heart Association

- The certification training consists of online interactive videos where the tester has to perform and demonstrate the neurological assessment

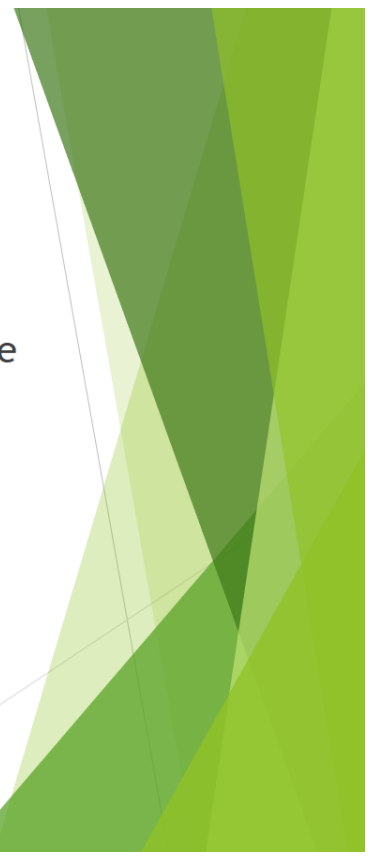




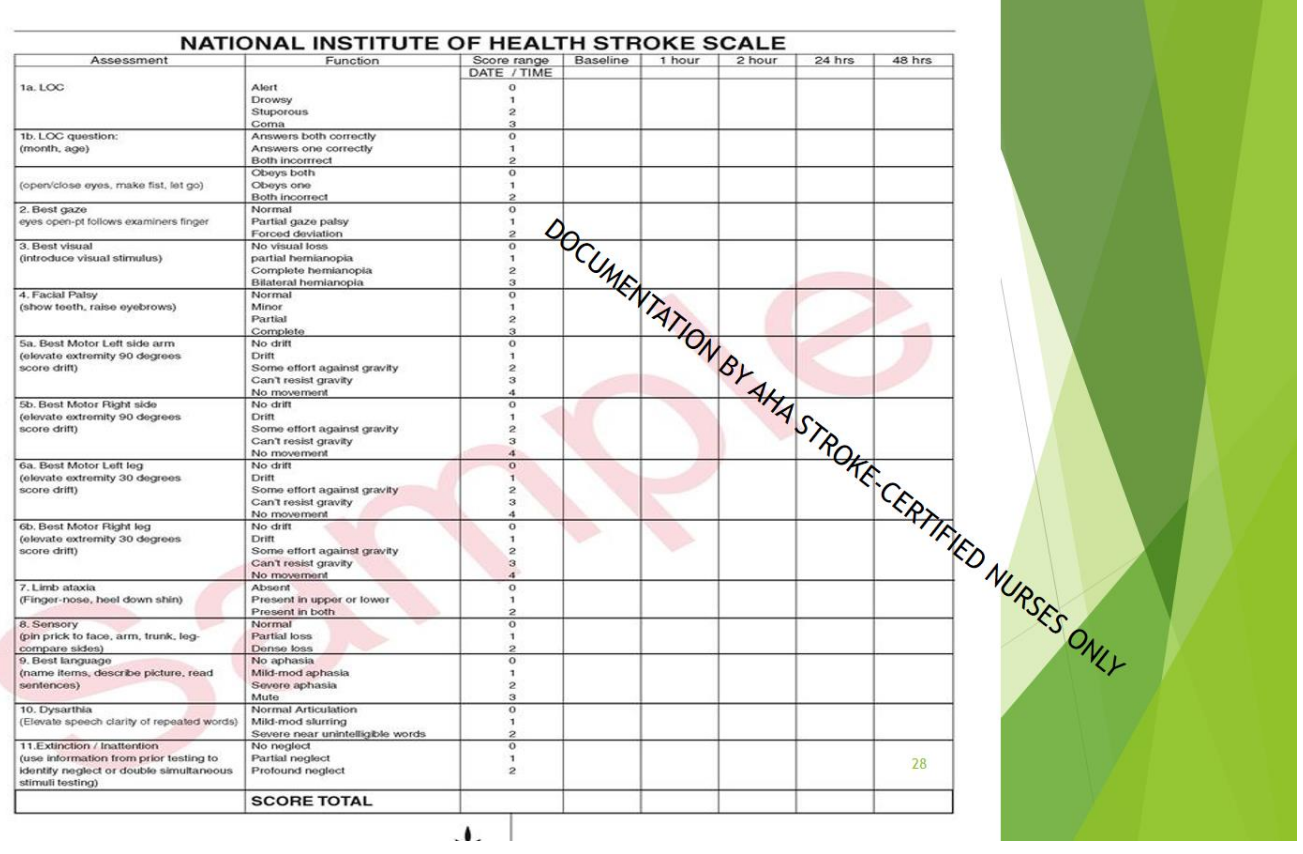

\section{Stroke Swallow Screen:}

must be done and completed before anything by mouth is given

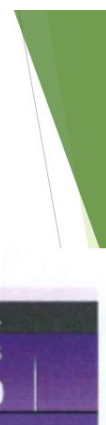

Complete Prior to Initation of Anything PO, Including Medication

Select an Approved Screening Diagnosis

Select Approved Credentials

Exclusion Criteria

Does the patient present as lethargic or with reduced alertness $\quad$ No

Foes the patient present with oxygen saturation $<90 \%$ RA or

直 Is patient having difficulty managing own secretions (drooling)

5 . Does patient/family report history of recent swallowing difficulty

\begin{tabular}{c|r}
\hline EDto Hos p-Admiss _. \\
\begin{tabular}{|c|r}
$4 / 23 / 16$ & $4 / 29 / 16$ \\
1700 & 1300 \\
\hline
\end{tabular}
\end{tabular}

Feeding and Swallowing Observation

9 Sit patient up in a 90 degree position

而 Give patient $3 \mathrm{oz}$. of water and instruct them to drink it all.

直 Does patient choke, cough, clear throat, sound wet, gurgly, or

Pass - Screen Complete

\begin{tabular}{|r|r|}
\hline RN:I hav.. \\
\hline No \\
\hline No \\
\hline No \\
\hline No \\
\hline Complet... \\
\hline Able to ... \\
\hline No \\
\hline PO diet ... \\
\hline
\end{tabular}




\section{Question}

What does the acronym F.A.S.T. stand for?

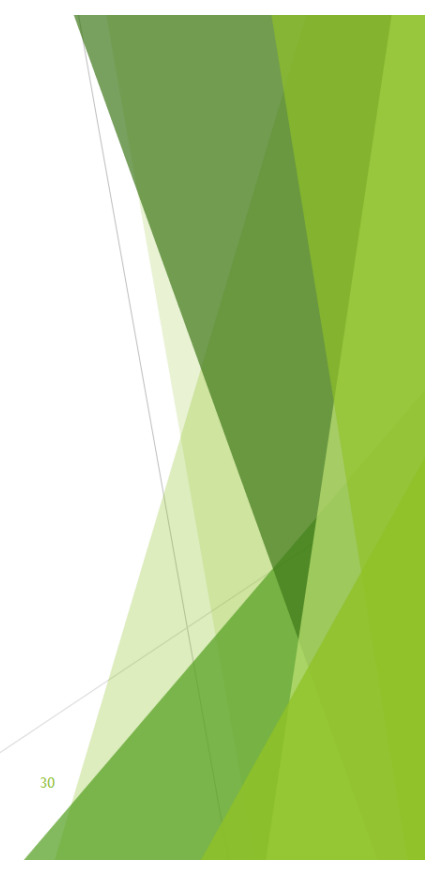

\section{Answer}

Facial droop, arm or extremity weakness, slurred speech and time

- If one or more of these symptoms are present then code stroke should be initiated
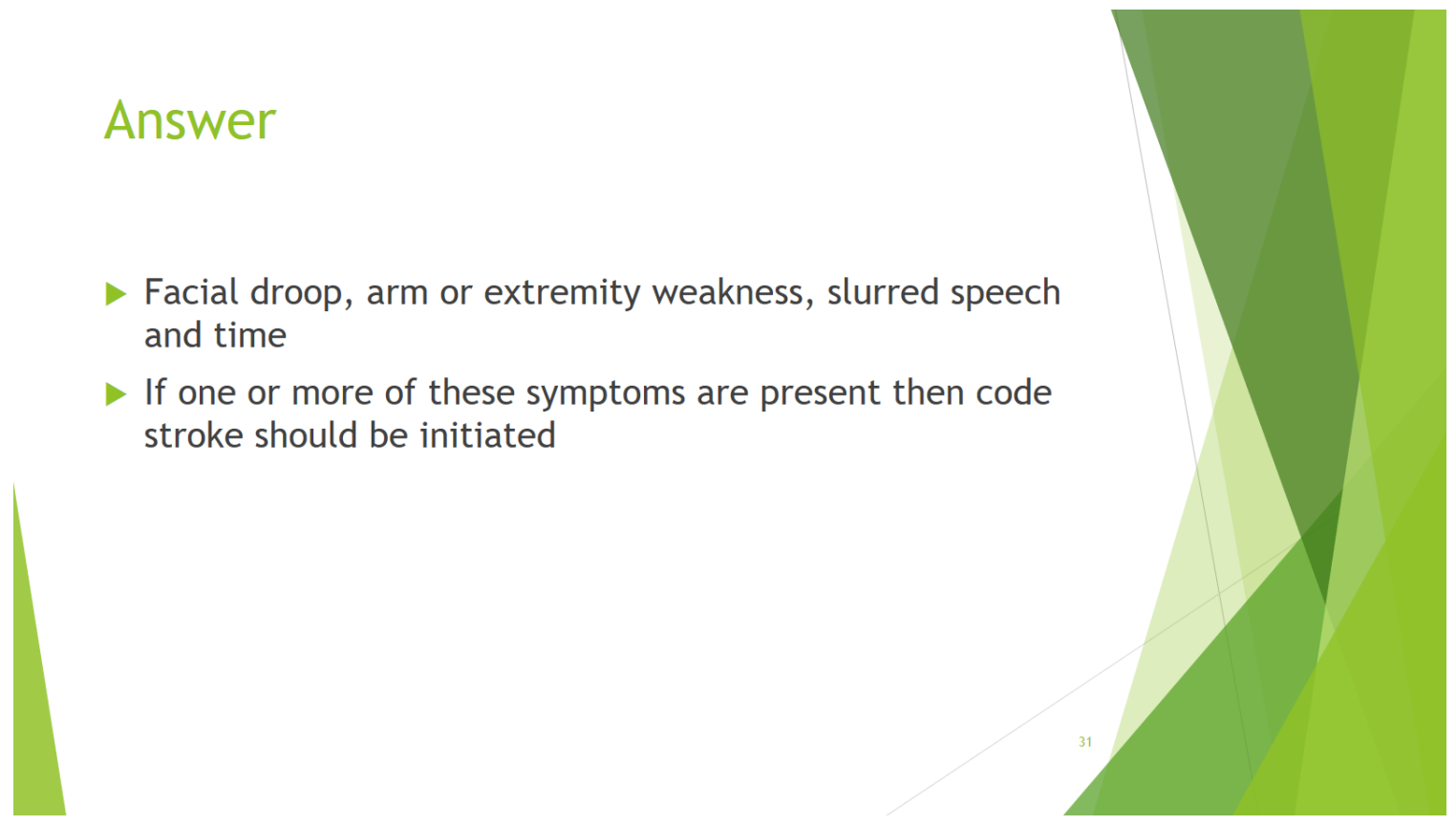


\section{Question}

- What interventions must be done during a code stroke but should not delay CT scan?

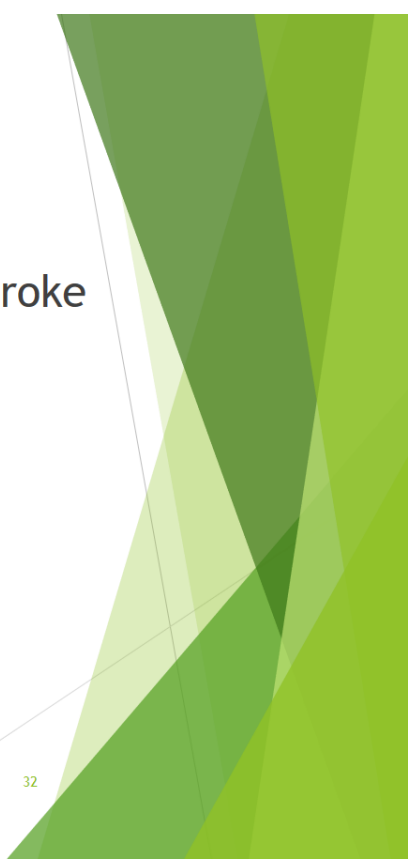

\section{Answer}

Blood sugar, T\&S, Coags, EKG, VS, neuro checks, pulse ox, document time of Last Known Well (LKW) and weight
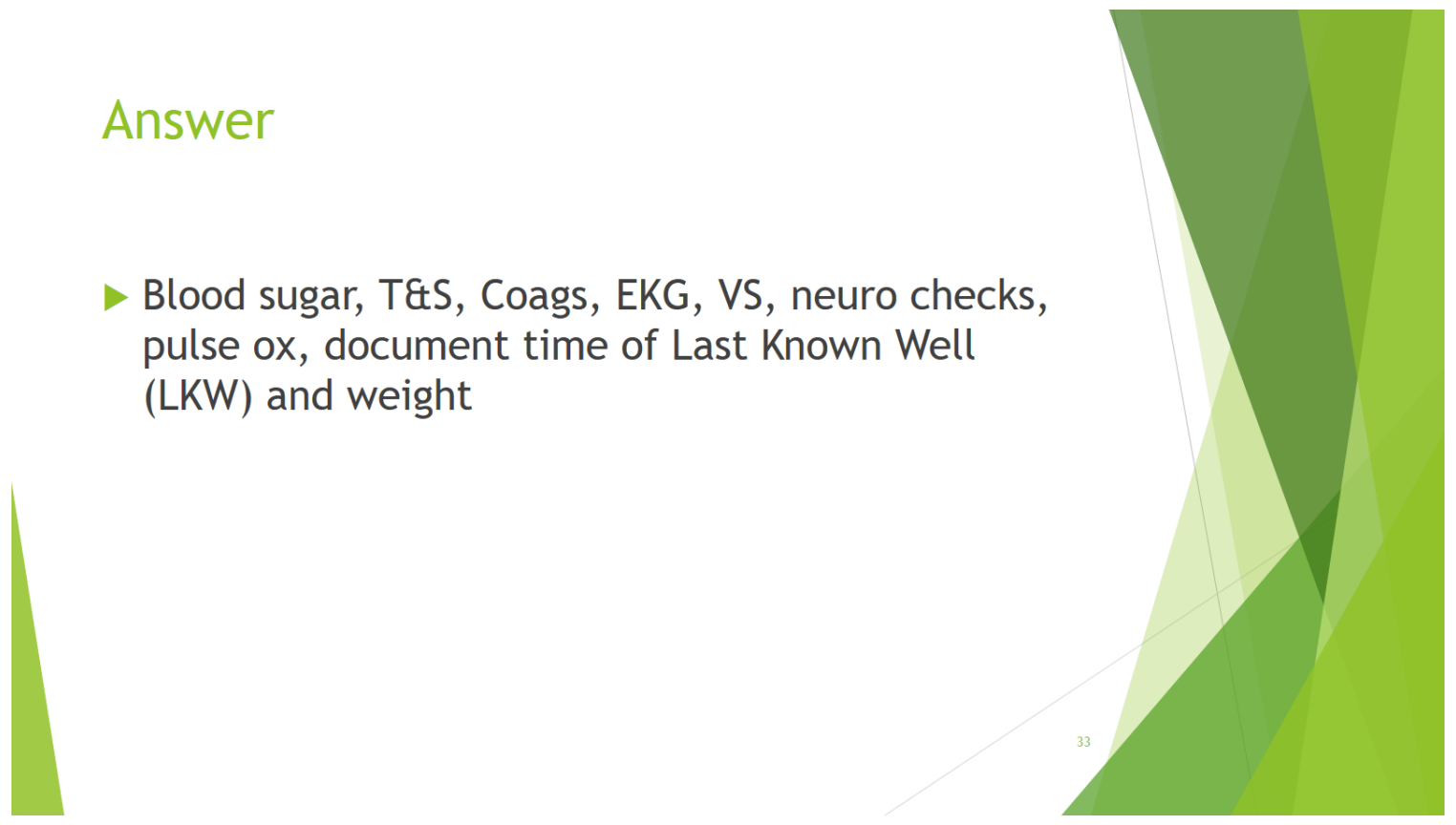


\section{Appendix B}

\section{Computer-based Module II}

Objectives: Identify possible stroke complications, state differences between Primary Stroke Center and Comprehensive Stroke Center, identify The Joint Commission stroke core measures and how they relate to patient care

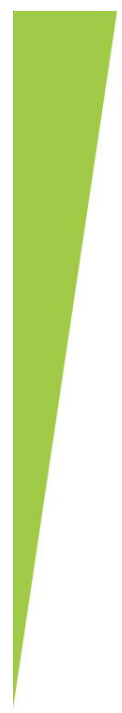

\section{Stroke Education} Module two

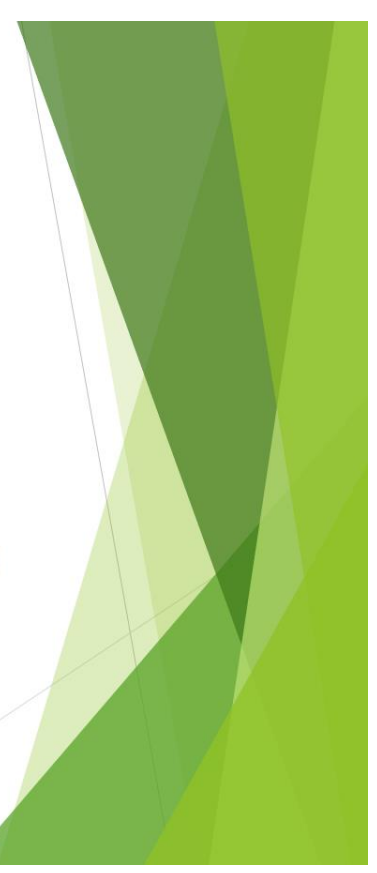




\section{Objectives}

- Identify and recognize possible stroke complications

- State differences between Primary Stroke Center (PSC) and Comprehensive Stroke Center

- Identify The Joint Commission (TJC) stroke core measures and how they relate to patient care

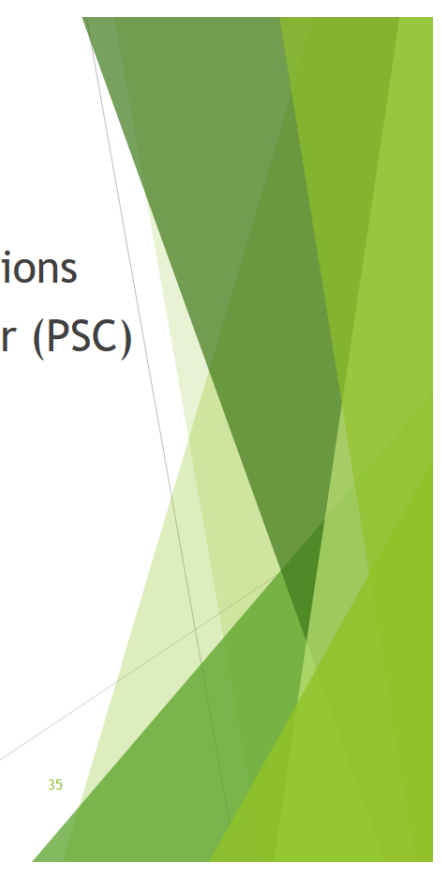

\section{Possible Early Post-Stroke Complications}

Cerebral edema

- Aspiration pneumonia

- UTI as a result of urinary catheter

- Seizures

$>$ DVT

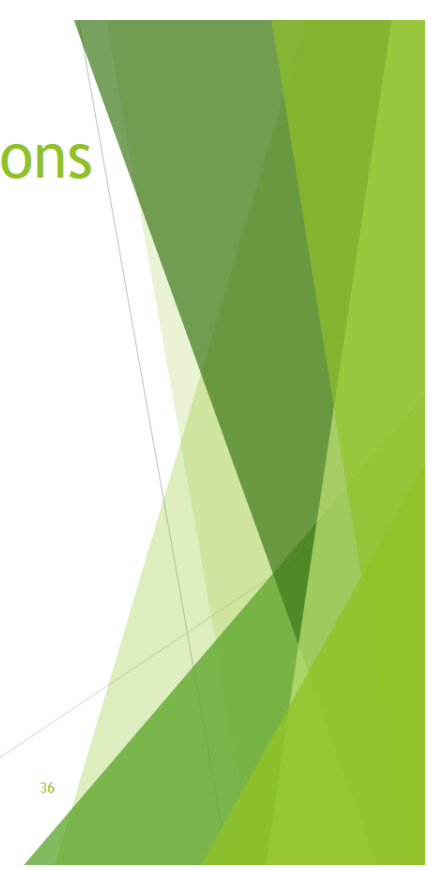




\section{Possible Long-term \\ Post-Stroke Complications}

- Depression

- Pressure ulcers

- Limb contractures

Cerebral vasospasm

-Hypo/hypertension

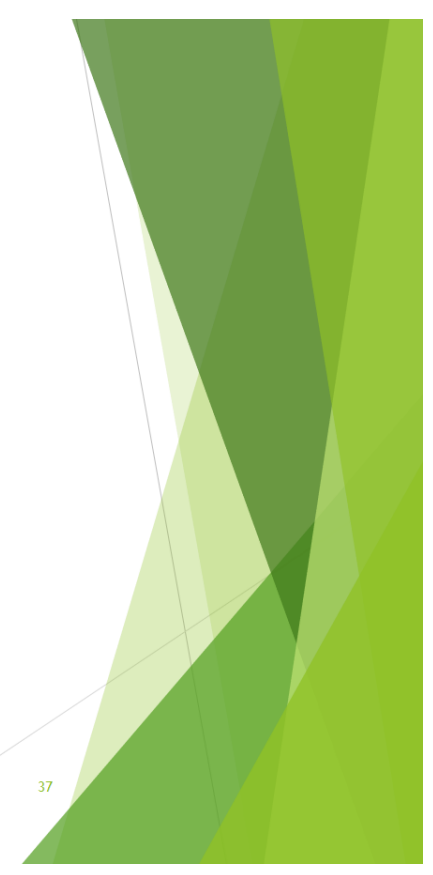

The Miriam Hospital Primary Stroke Center: Requirements

A dedicated stroke-focused program

- Staffing by qualified medical professionals trained in stroke care

- Individualized care to meet stroke patient needs

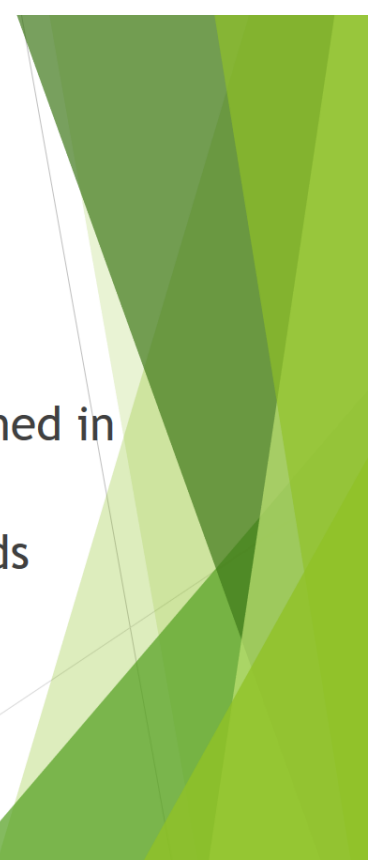




\section{The Miriam Hospital Primary Stroke Center: Requirements}

- Hospital collects stroke-treatment performance data

- Hospital collects stroke team performance data

- Uses data to assess and improve quality of care for stroke patients

The Miriam Hospital Primary Stroke Center: Requirements

- Patient involvement in their hospital care

- Coordination of post-discharge patient self-care based on recommendations of the Brain Attack Coalition and guidelines published by the American Heart Association/American Stroke Association or equivalent guidelines

- Streamlined flow of patient information while protecting patient rights, security and privacy

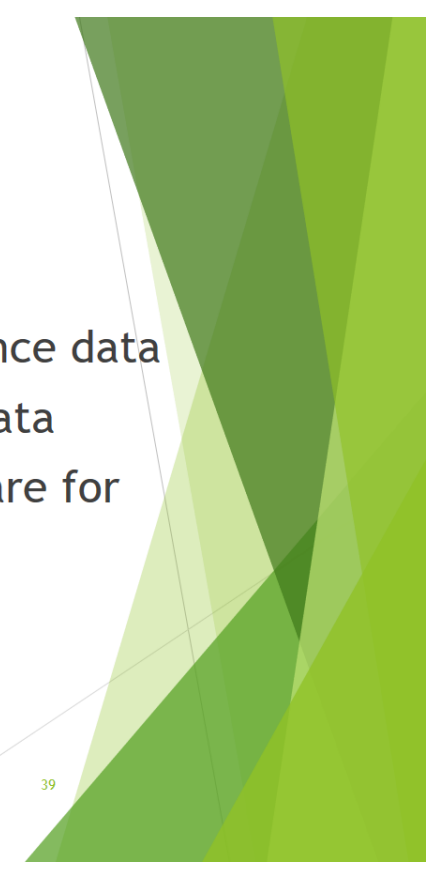




\section{Comprehensive Stroke Center (Rhode Island Hospital)}

- Significant resources to provide advanced care $\checkmark$ Neuro ICU

- Advanced imaging capabilities

- Meet requirements for caring for patients diagnosed with subarachnoid hemorrhage

Comprehensive Stroke Center (Rhode Island Hospital)

Coordinates post-hospital care

-Utilize peer review process to evaluate care

Analyze performance on 16 required measures

Participates in stroke research

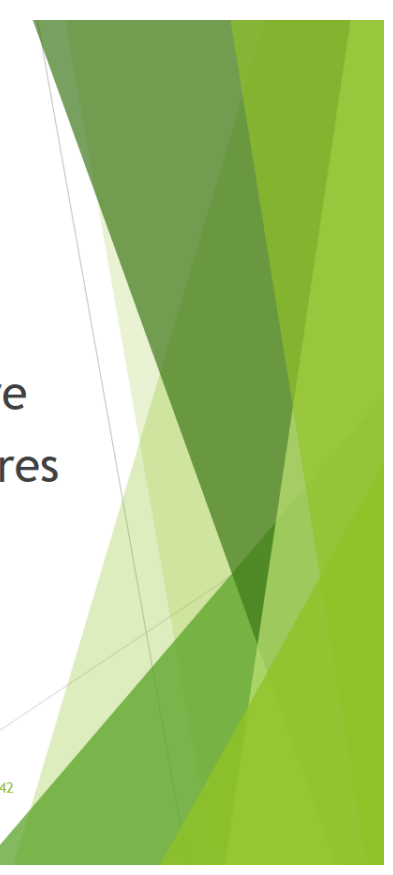




\section{Comprehensive Stroke Center: Endovascular Thrombectomy}

- Comprehensive Stroke Center only

- Procedure used in ischemic stroke

Mechanically removes thrombus (percutaneous catheterization)

- Re-establishes blood flow

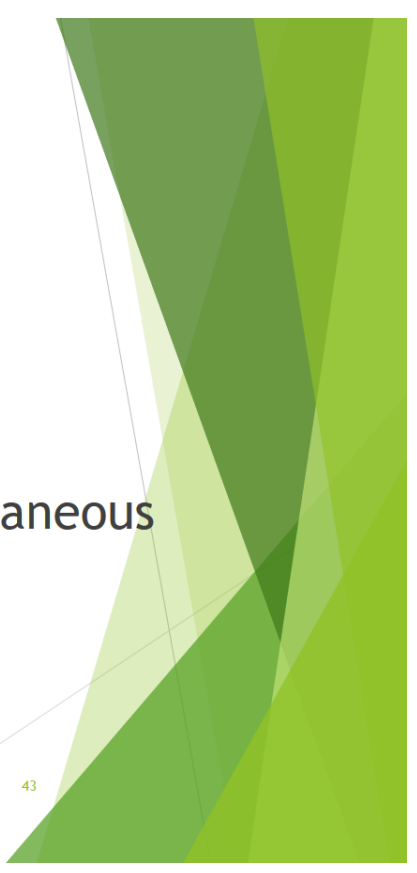

Comprehensive Stroke Center Endovascular Thrombectomy

- Endovascular thrombectomy takes minutes vs Alteplase (tPA) which can take up to 2 hours

- Limitations: catheter can only remove thombi from large cerebral vessels

- Alteplase and thrombectomy are treatments done simutaneously

- $60 \%$ of patients have better clinical outcomes over Alteplase alone

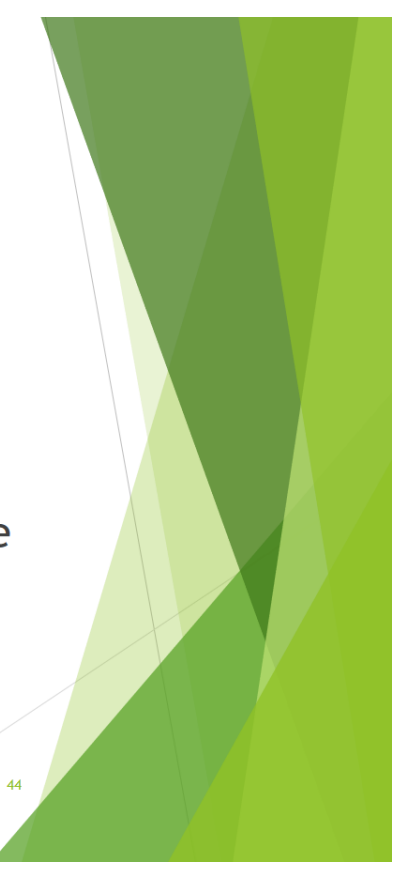




\section{The Joint Commission} Stroke Core Measures (STK)

\section{Joint Commission Stroke (STK) Core Measures for All Strokes}

- STK-1: venous thromboembolism prophylaxis

- STK-2: discharged on antithrombotic therapy

- STK-3: anticoagulation therapy for atrial fibrillation/flutter

- STK-4: thrombolytic therapy

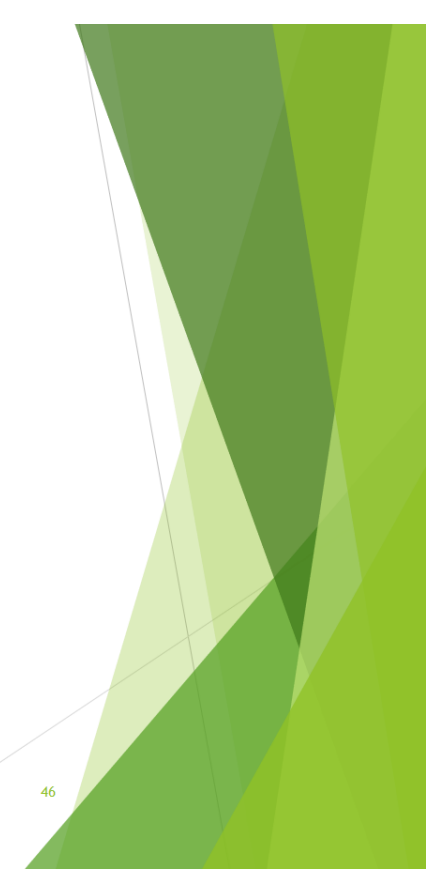




\section{Joint Commission Stroke (STK) Core Measures}

STK-5: antithrombotic therapy by the end of hospital day 2

- STK-6: discharged on statin medication

- STK-8: stroke education

- STK-10: assessed for rehabilitation

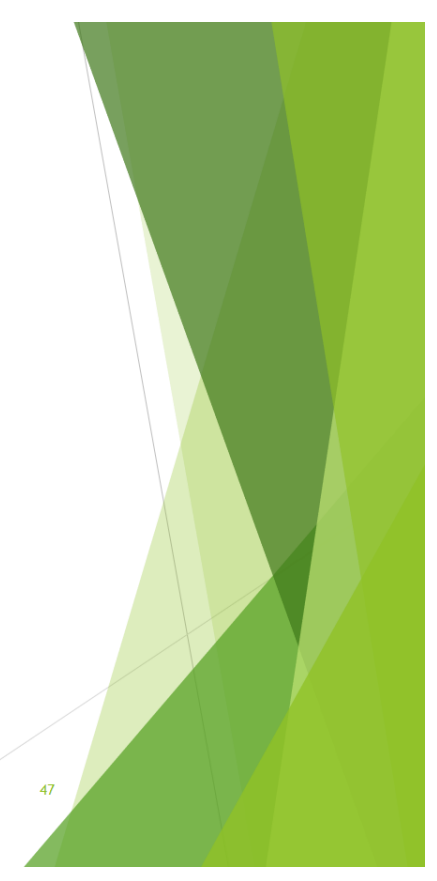

\section{Case study: Ms. J}

78 year old African American woman is found down at home in her bedroom. Her daughter finds her at 0730 . She was brought to the emergency room by EMS. The daughter reports slurred speech and new dominant hand weakness. A CT scan showed an ischemic stroke. She was given Alteplase and admitted to the ICU 24 hours. After transfer to 3 East, it is reported to you that the patient has garbled speech, right sided hemiplegia, is able to follow commands with left side, and able to write answers to questions. What are you going to make sure is part of her treatment plan?

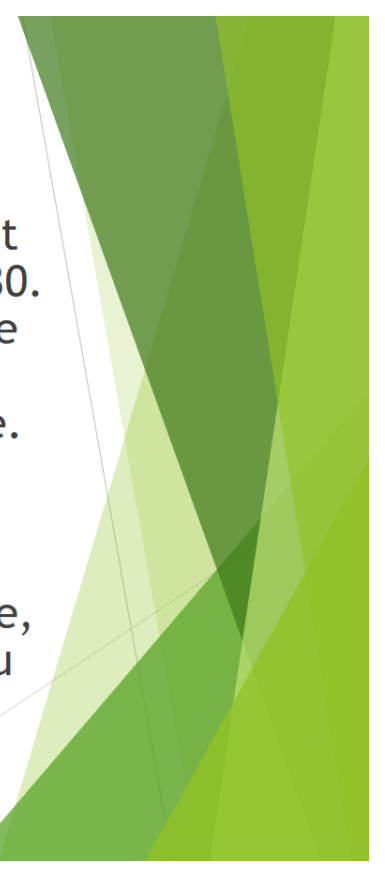




\section{Plan of Care for Ms. J}

- Plan of care must include all aspects of The Joint Commission Stroke Core Measures

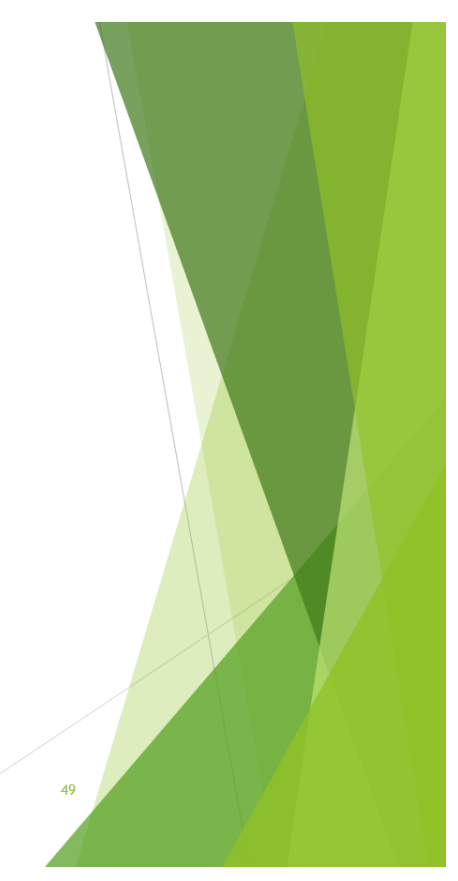

\section{STK-1: Venous Thromboembolism Prophylaxis}

- All patients diagnosed with stroke should have DVT prophylaxis ordered

- If DVT prophylaxis is contraindicated then LIP must document

- This is required due to high risk of DVT in stroke patient

- Patients need anticoagulation or antiplatelet therapy in addition to aspirin therapy 


\section{STK-1: Venous Thromboembolism Prophylaxis Rationale}

Stroke patients are at high risk for deep vein thrombosis (DVT) and pulmonary embolism (PE)

- A PE occurs in approximately $10 \%$ of stroke patients. DVT and PE most likely happen in the first 3 weeks after stroke. $\mathrm{PE}$ develops from venous thrombi in a paralyzed limb secondary to stroke. Early mobilization, external compression devices and use of antithrombotic agents are all sufficient interventions that meet the requirement of this measure

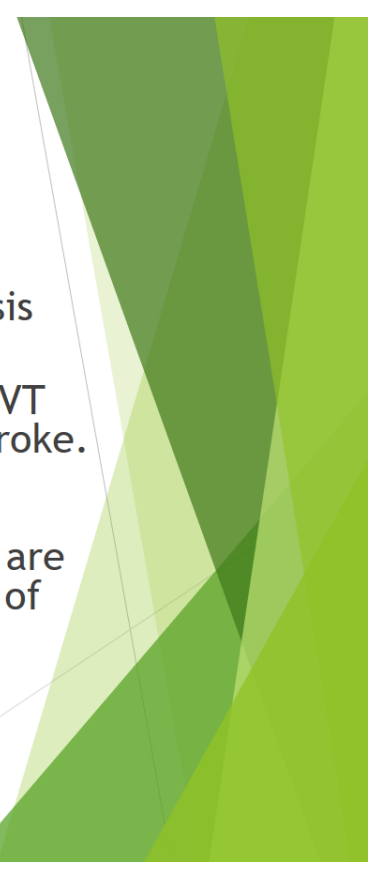

\section{STK-2: Discharged on Antithrombotic Therapy}

- Patients diagnosed with ischemic stroke need to be prescribed a form of antithrombotic therapy

- Research has shown that antithrombotic therapy helps decrease the high risk of stroke reoccurance

- This is especially important with patients whose stroke is attributed to a cardioembolic event 


\section{STK-5: Antithrombotic Therapy by End of Hospital Day 2}

Antithrombotic therapy must be started within 2 days of acute stroke in patients

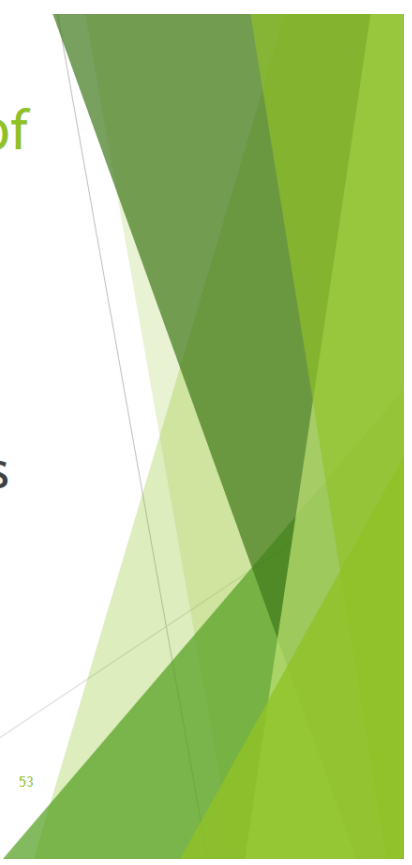

\section{STK-2 and STK 5: Rationale}

Antithrombotic therapy decreases risk of stroke recurrence, as well as morbidity and mortality

- Aspirin Therapy Contraindication:

- Current data from the AHA indicates a small significant increase in mortality and unfavorable patient outcomes, including stroke recurrence, when aspirin is taken after a stroke

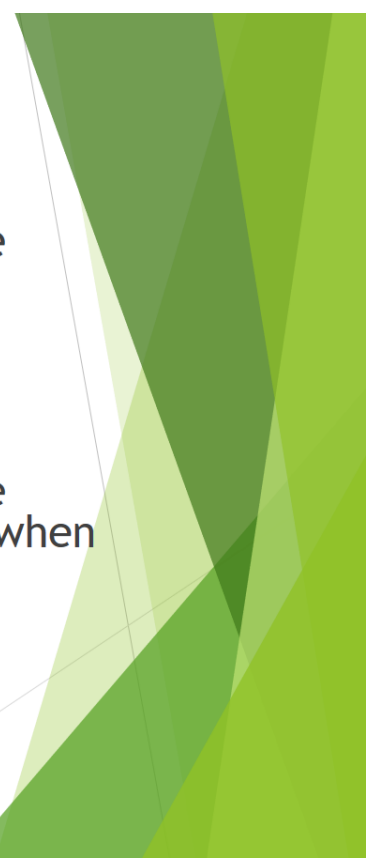




\section{STK-3: Anticoagulation Therapy for Atrial Fibrillation/Flutter}

Patients with atrial fibrillation/flutter must be discharged with anticoagulation therapy

- The risk of stroke in patients with atrial fibrillation/flutter increases with age

- Studies have shown a decrease in stroke occurrence by as much as $68 \%$ in patients treated with warfarin

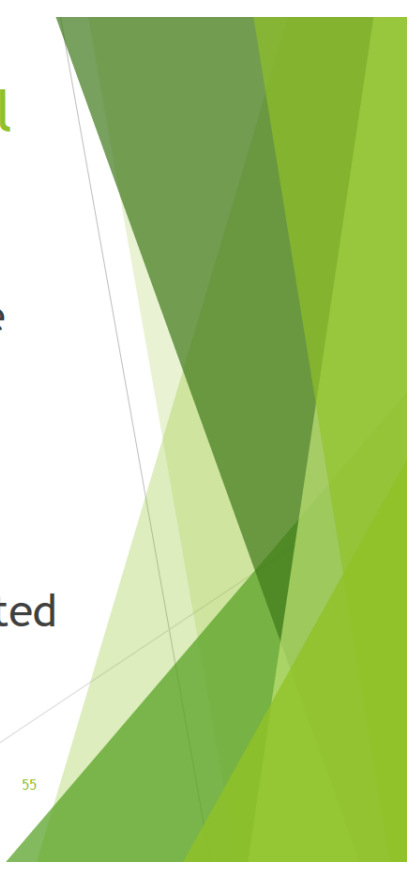

\section{STK-4: Thrombolytic Therapy}

- Alteplase has proven to be highly effective in patients who are carefully screened and who meet specific criteria related to medical history, time of stroke onset, and symptoms of ischemic stroke

- The recommended medication is IV tissue plasminogen activator (Alteplase) tPA. Maintaining (Alteplase) tPA administration compliance within the time requirements is vital for achieving and sustaining stroke center certification. Shorter needle to door time is associated to improved outcomes, decreased complications and shorter length of stay

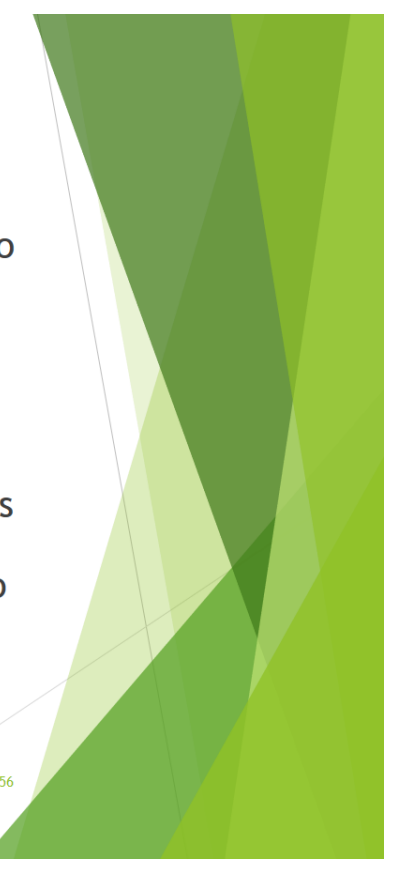




\section{STK-4: Thrombolytic Therapy Rationale}

- Use of thrombolytic therapy minimizes disability by $30 \%$

- Patients who delay thrombolytic therapy by as little as 10 minutes are at an increased risk for disability

> $40 \%$ of patients treated with Alteplase were discharged to home

> $38.7 \%$ treated with Alteplase ambulated independently at discharge

- The required administration time is within 60 minutes of arriving to emergency room or time that code stroke is initiated on the inpatient floor. Alteplase (Tissue Plasminogen Activator-tPA) is the thrombolytic given to stroke patients

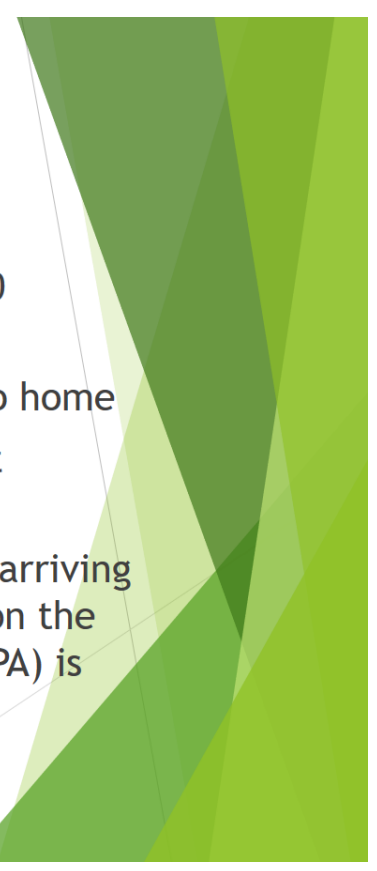

\section{STK-6: Discharged on Statin Medication}

- Increased serum lipid levels have proven to be a risk factor for both stroke and cardiac events

- Patients with a low-density lipoprotein (LDL) cholesterol level equal-to or greater that $100 \mathrm{mg} / \mathrm{dL}$ should be started on and discharged on a statin

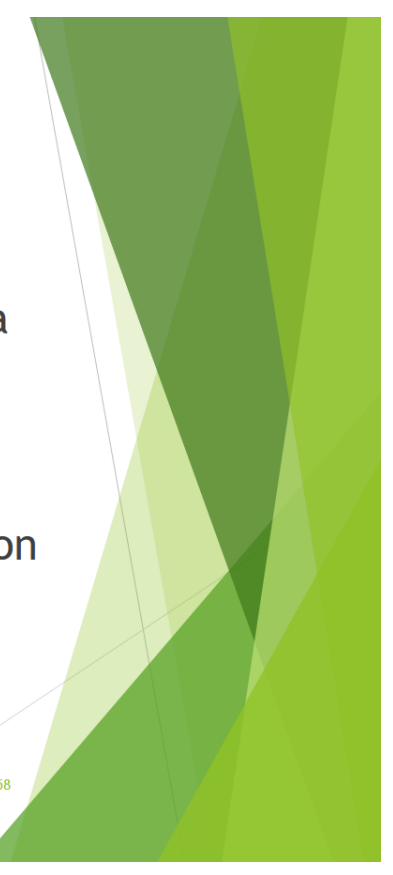




\section{STK-6: Discharge on Statin Medication Rationale}

- Statins, cholesterol lowering medications, slow the progression of atherosclerosis, stabilizes plaque, reduces risk of rupture and risk of arterial thrombus therefore improving prognosis

- Statins reduce the risk of stroke by $30 \%$. All patients with established cerebral disease should be prescribed a statin in conjunction with lifestyle changes

(Goldszmidt \& Caplan, 2010) (Elkind, 2009)

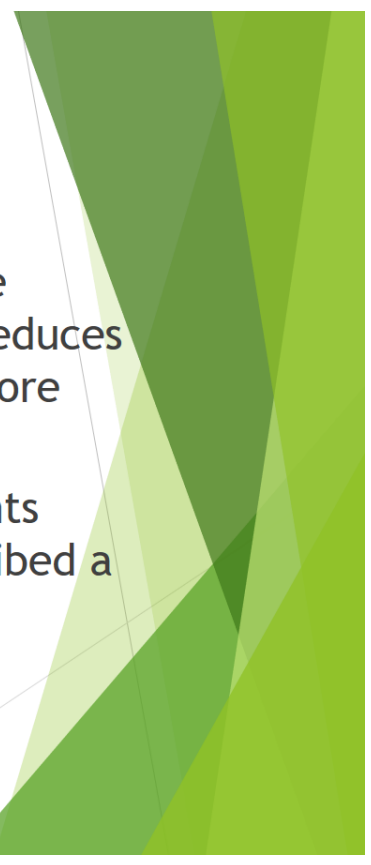

\section{STK-8: Stroke Education}

- All patients with stroke diagnosis must receive stroke education

- Preventive health education on the control of risk factors is vital to recovery and prevention of reoccurrence

- Education should focus on identifying symptom onset and seeking immediate medical care.

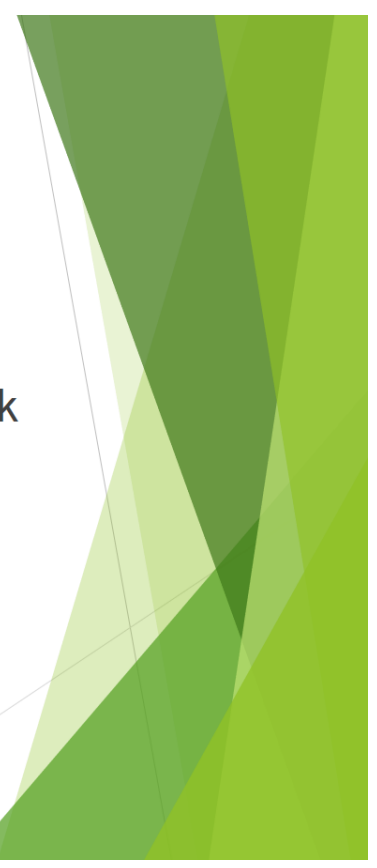




\section{STK-8: Stroke Education Rationale}

- Patients are educated regarding importance of follow-up care and medication compliance

- Data demonstrates that the public's knowledge of onset of stroke symptoms remains poor

- Less than half of patients who call EMS recognize their symptoms for possibly being caused by a stroke

- In order to ensure the likelihood of decreased disability from stroke the first step is early recognition

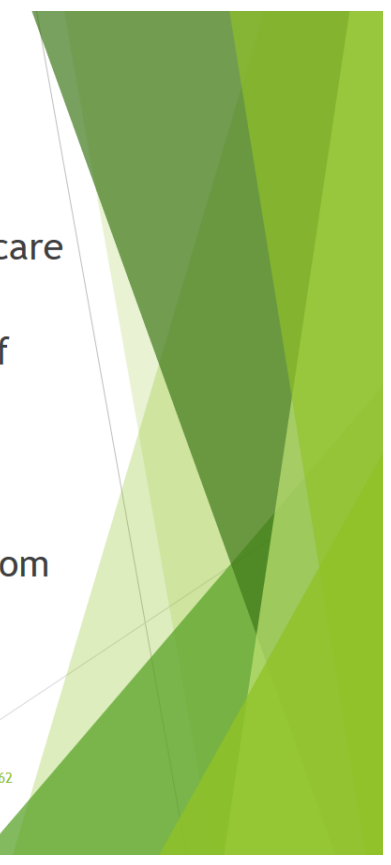

\section{STK-8: Stroke Community Education Rationale}

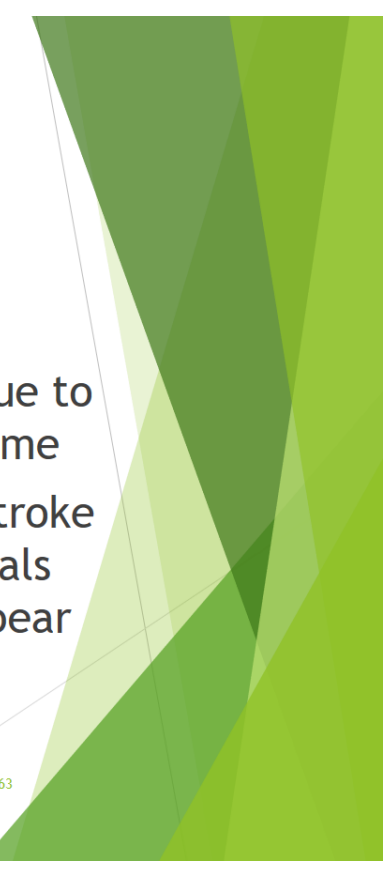

$>90 \%$ of patients who seek treatment for stroke symptoms are not eligible for fibrolytic therapy due to not seeking treatment within the desired time frame

- This delay is due to lack of lack of awareness of stroke symptoms. It is imperative to educate all individuals to seek treatment immediately if symptoms reappear 


\section{STK-10: Assess for Rehabilitation}

- Rehabilitation is immediate and ongoing. As soon as the patient is stable they should be assessed by rehab.

- Rehab begins as soon as possible to increase function and to improve quality of life

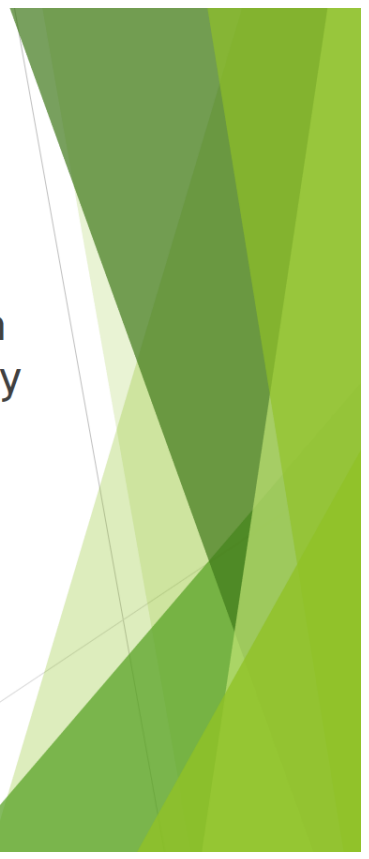

\section{STK-10: Assessed for Rehabilitation Rationale}

- Stroke patients are at risk for serious complications caused by immobility

- Early ambulation and physical therapy decreases stroke disability

- A Very Early Rehabilitation Trial for Stroke (AVERT) was a large randomized controlled trial that mobilized patients within the first 24 hours after stroke. The pilot program demonstrated that early mobilization of stroke patients resulted in fewer complications such as DVT, Pneumonia and pressure ulcers

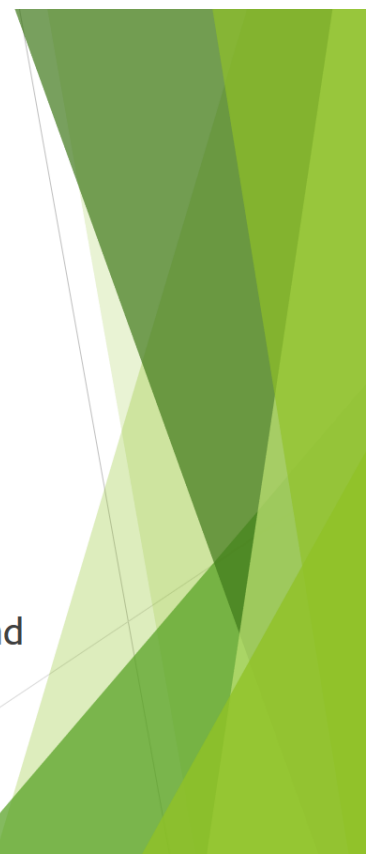




\section{Question}

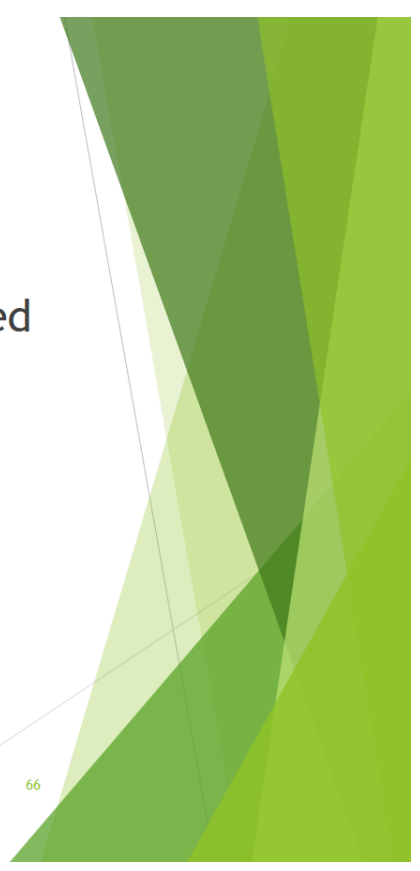

\section{Answer}

- Lipid panel: increase levels of cholesterol correlates directly with increased risk for stroke

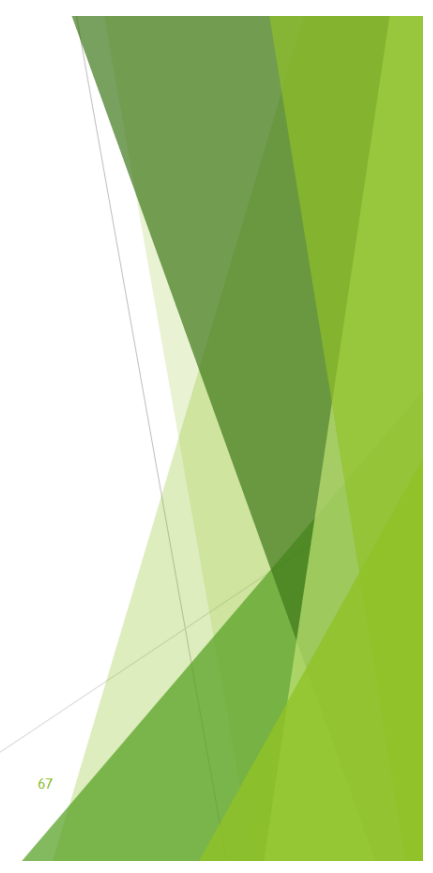




\section{"Time is Brain"}

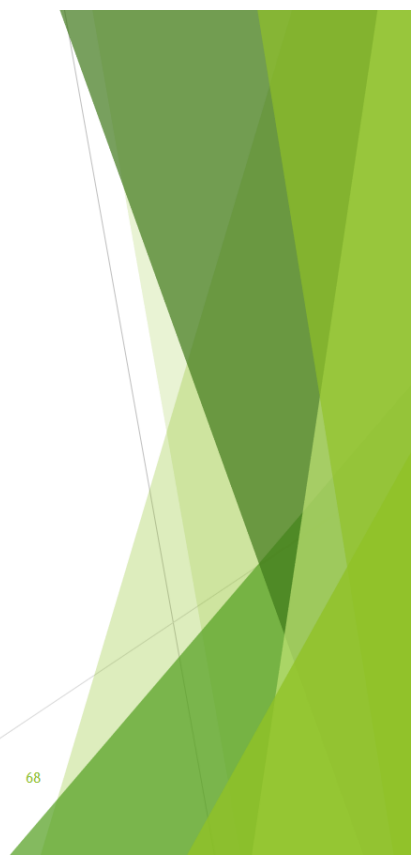


Appendix C

Computer-bases Module III

Objectives: Define American Heart Association: Get with the Guidelines (GWTG), and describe appropriate interventions to do during a code stroke

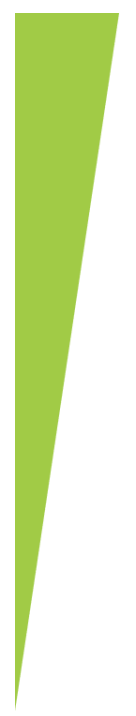

\section{Stroke Education}

Module three 


\section{Objectives}

- Define American Heart Association: Get with the Guidelines (GWTG)

- Describe appropriate interventions to do during a code stroke

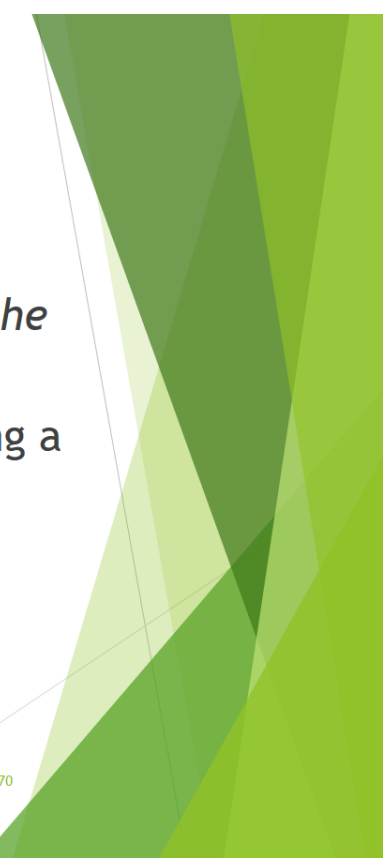

\section{American Heart Association (AHA) Get With The Guidelines (GWTG)}

Distinct from TJC core measures, the AHA program, Get with The Guidelines - Stroke (GWTG), is a hospital program for improving stroke care by promoting consistent adherence to the most current scientific treatment guidelines.

- From 2003 to 2007 a study of 322,847 hospitalized stroke patients in 790 US academic and community hospitals voluntarily participated in GWTG- stroke program showed significant improvement in stroke care.

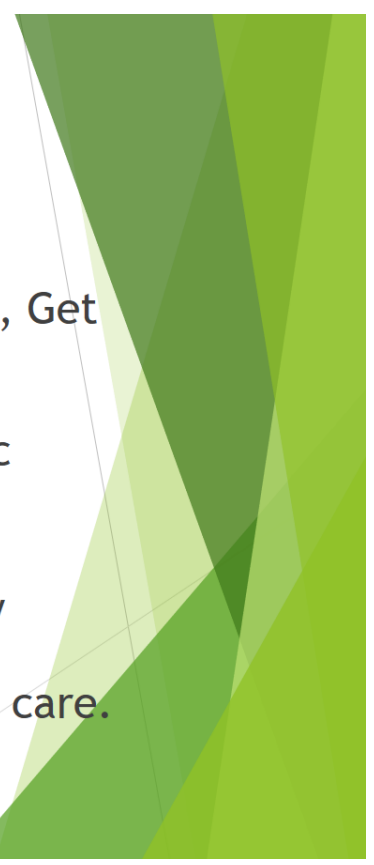




\section{American Heart Association (AHA) Get With The Guidelines (GWTG) Awards}

- The goal of the AHA program is to ensure positive patient outcomes when evidence-based medicine is practiced

- Silver, Gold, Silver Plus and Gold Plus Award-winning "Get With The Guidelines-Stroke" hospitals are honored at national recognition events during the International Stroke Conference

- Silver, Gold, Silver Plus and Gold Plus Award-winning "Get With The Guidelines-Stroke" hospitals are listed by name in advertisements that appear annually in the journal Stroke and in the "Best Hospitals" issue of U.S. News \& World Report

- All award-winning hospitals are provided marketing materials to use to announce their achievements locally

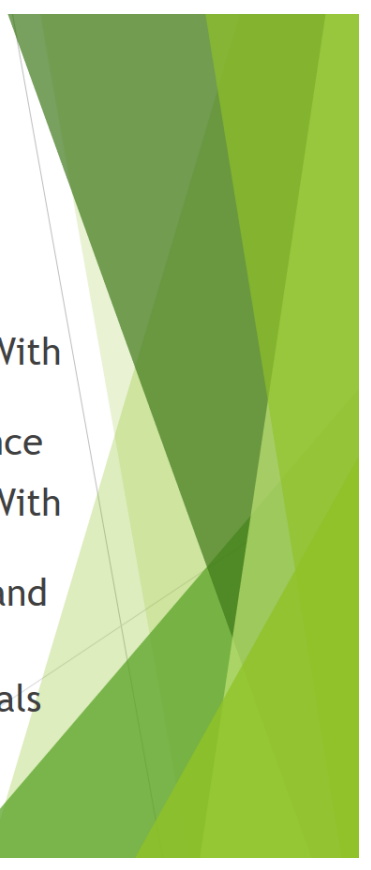

\section{Code Stroke and Beyond}

During hourly rounding on one of your patients, you note that her right eye is drooping. What do you do?

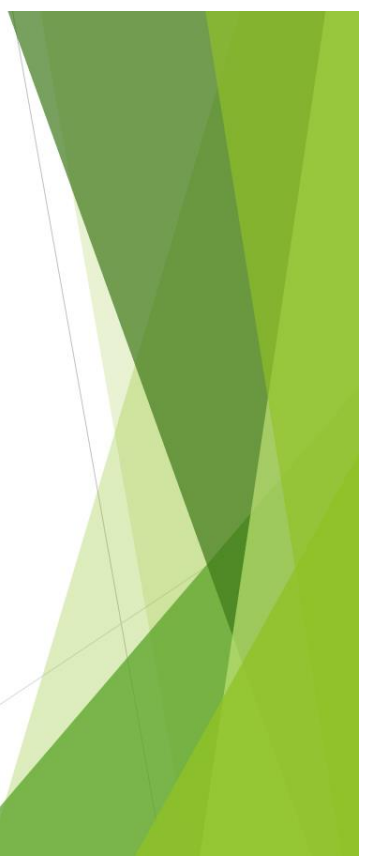




\section{Code Stroke and Beyond}

\section{Think F.A.S.T.}

\section{Code Stroke and Beyond}

Face: drooping on one side, or numbness

Arms (or legs): Weakness or numbness on one side (examples: weakened grasp, unsteady gait)

Speech: Slurred or trouble swallowing

Time: Document time and act quickly - Time is Brain
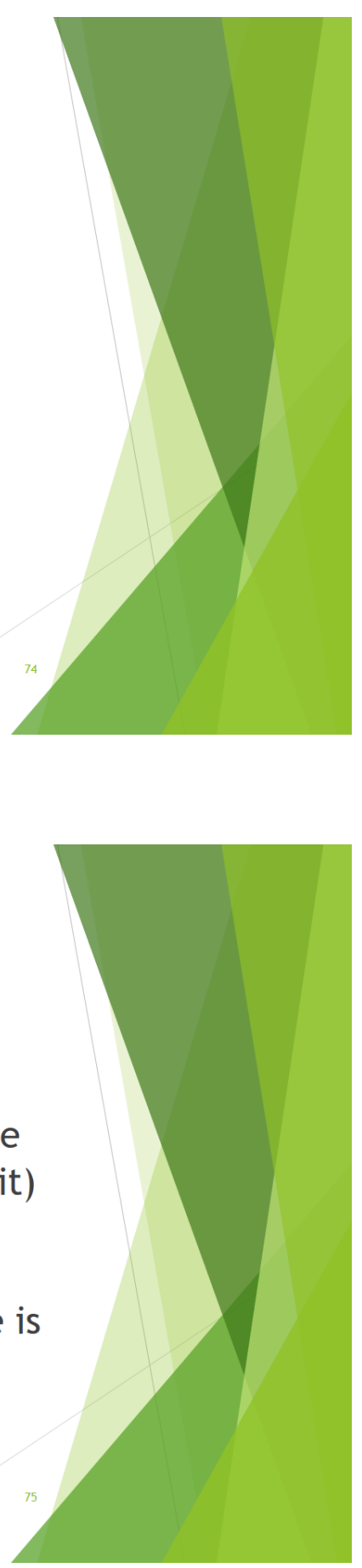


\section{Code Stroke and Beyond}

- Call 3-5111 and ask operator to activate Rapid Response Team and code stroke

- Page the responsible MD/PA/NP - be sure to page LIP responsible for the patient, even if other LIPs respond to the RRT and code stroke page

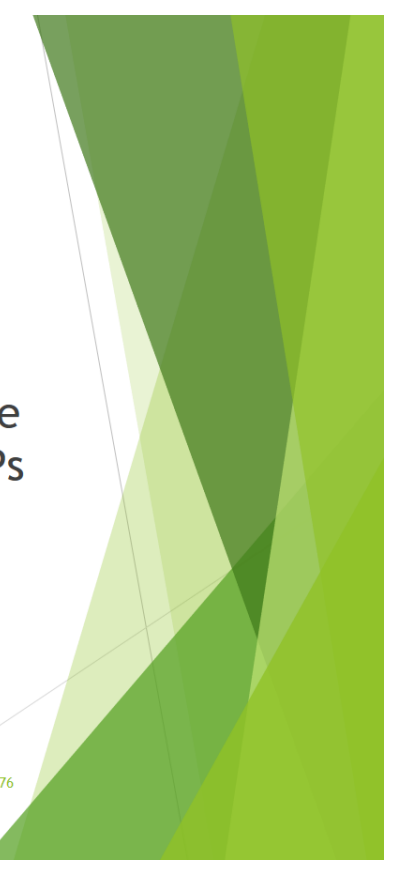

\section{Code Stroke and Beyond}

- Obtain Last Known Well (KNW- The time at which the patient was last known to be without the signs and symptoms of the current stroke or at his/ her prior baseline), weight, blood sugar, apply portable cardiac monitor and prepare to transport to CT scan

- Perform Neuro and vital signs- document of RRT downtime form

- If any of the above can't be done- document

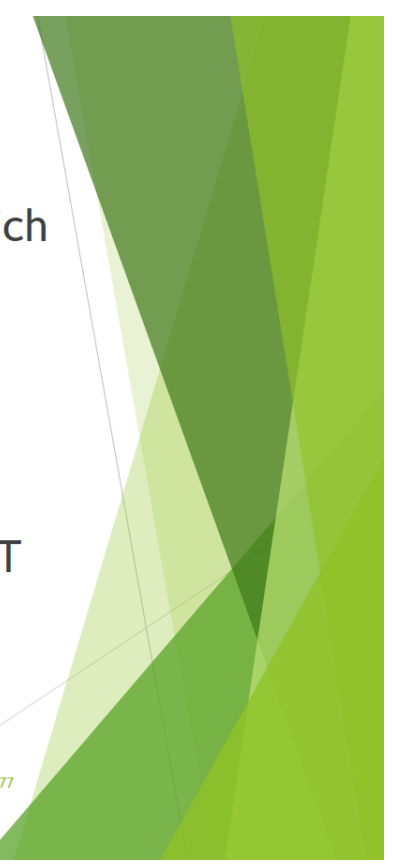




\section{Code Stroke and Beyond}

- In order to provide highest quality of care the following time metric must be adhered to

- 25 minutes: Time of code stroke to CT scan must happen in 25 minutes

$\checkmark 20$ minutes: CT scan must be read within 20 minutes

- 60 minutes: Alteplase must be given within 60 minutes of symptom onset

- Patients who receive Alteplase are then transferred to ICU, RN must travel with patient to CT Scan

\section{Code Stroke and Beyond}

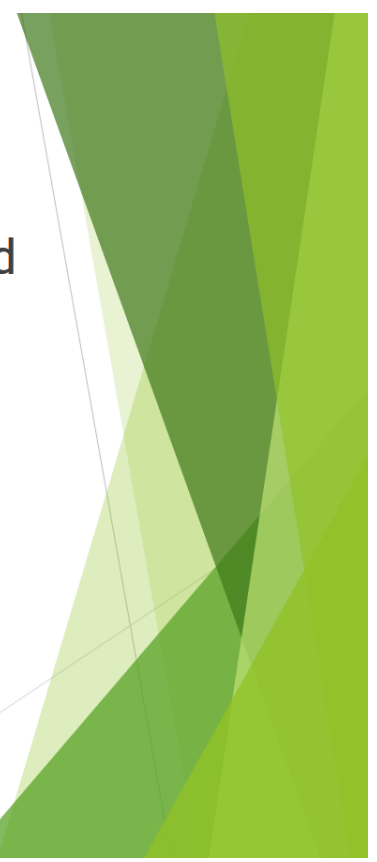

The following should also be done but should not delay CT Scan

obtain EKG

- Oxygen 2 liters via nasal cannula if oxygen saturation is less the $92 \%$

- STAT Labs
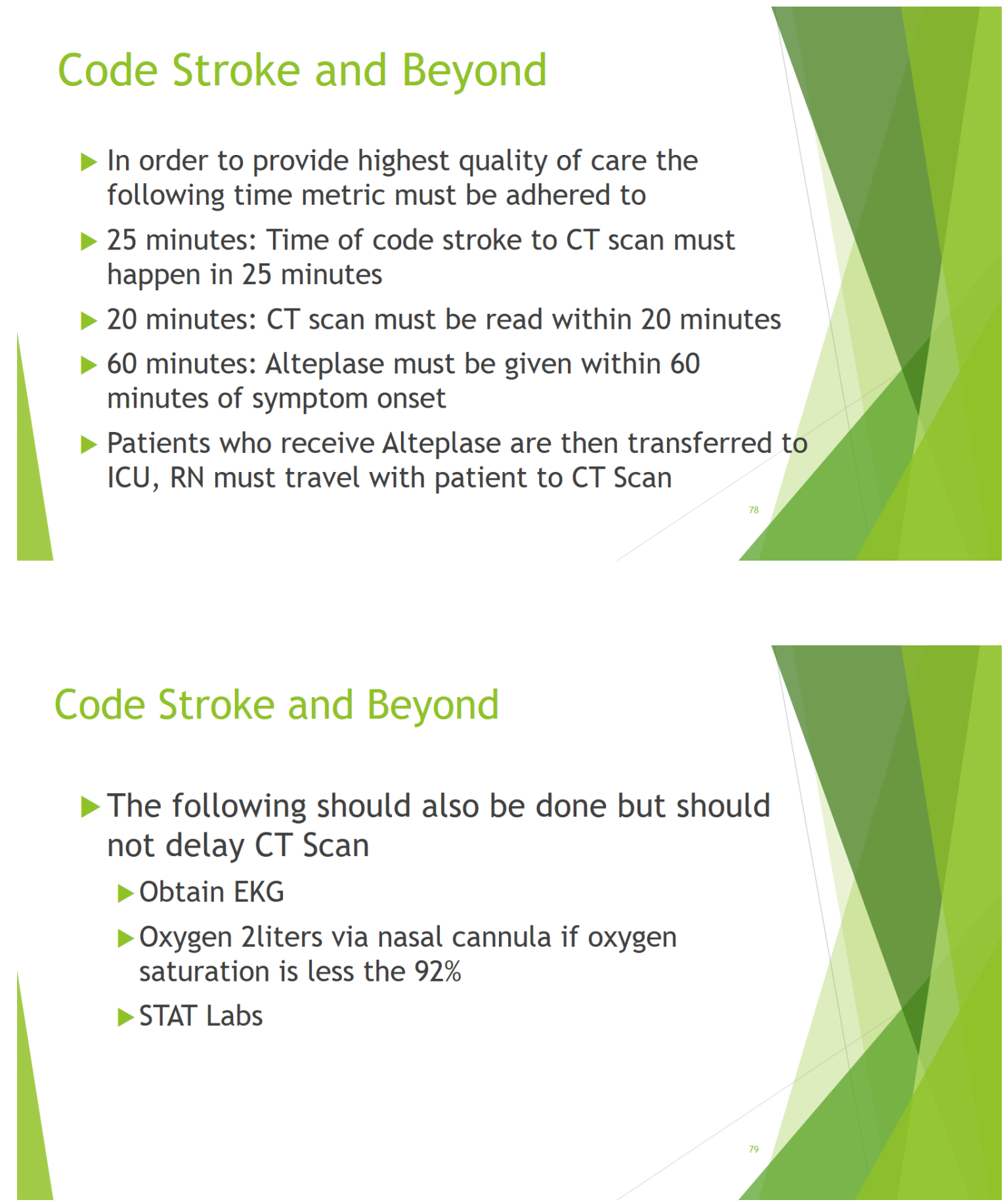

STAT Labs 


\section{Code Stroke and Beyond}

- CBC, PT, PTT, INR, Type and Screen, Pregnancy test, if applicable

- Complete Significant Event note

- Ordering Provider / Nursing Supervisor/Stroke Coordinator must complete NIH Stroke Scale on all code stroke patients and before Alteplase administration

- ICU nurse remains with patient until it is determined if Alteplase will be given

\section{Code Stroke and Beyond}

Post code stroke

- All patients should be NPO until Stroke Swallow Screen is complete and aspiration risk assessed

- Individualized patient education with close attention to appropriate risk factors

- Every patient must be assessed by rehab

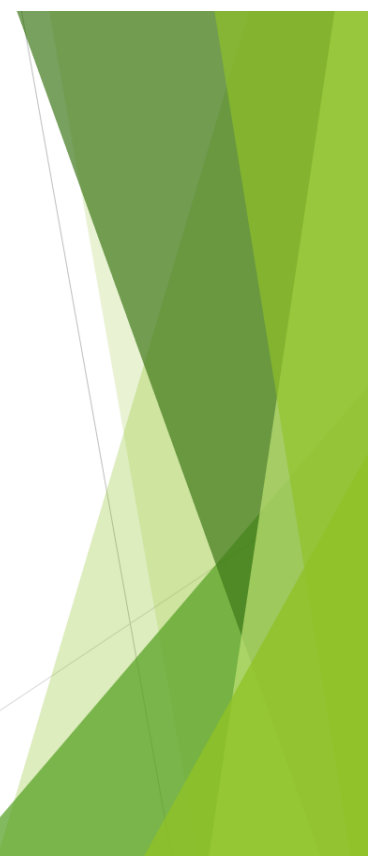




\section{Code Stroke and Beyond}

- What interventions are completed during a code stroke?
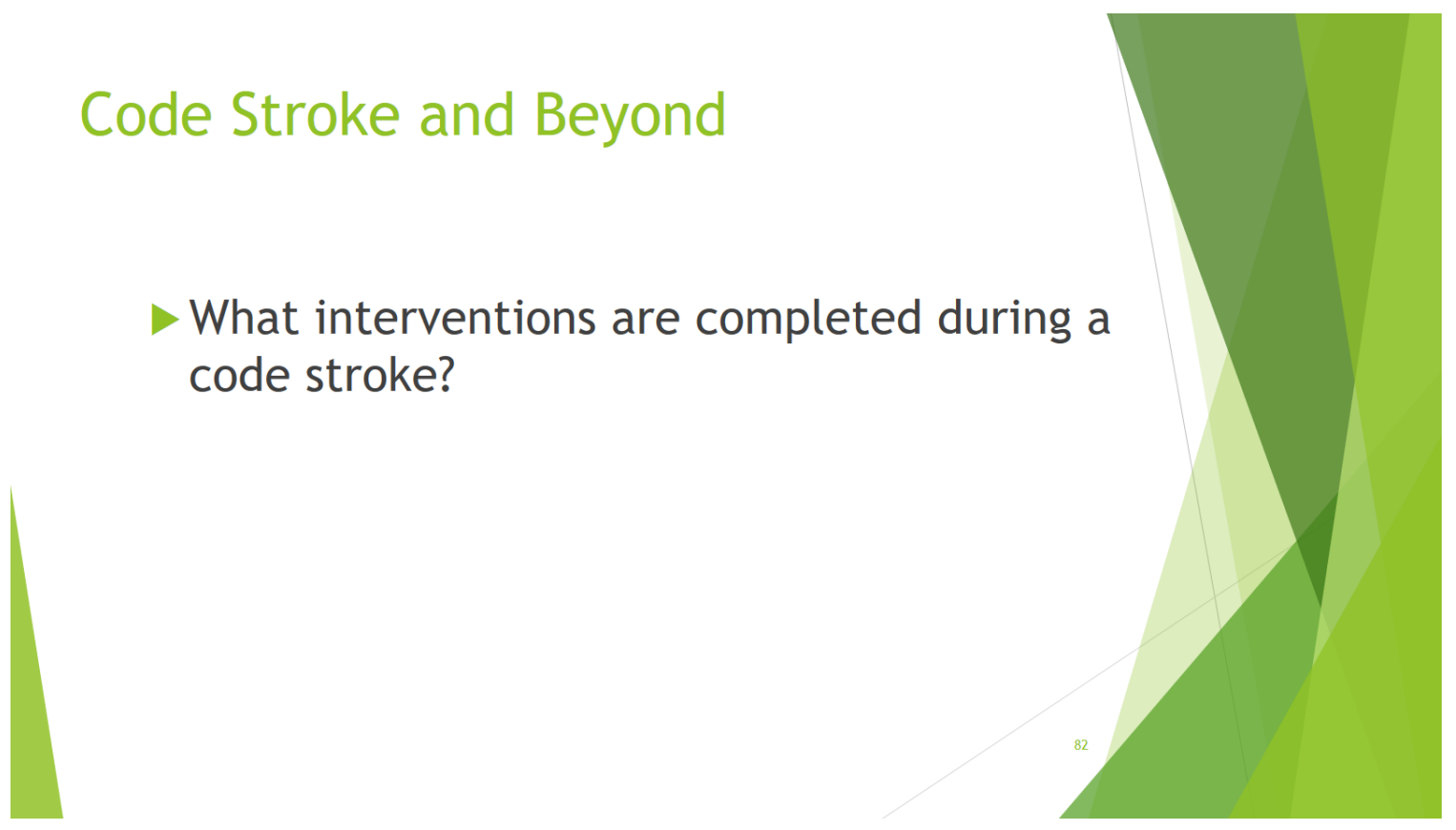


\section{Appendix D}

Objectives: Describe the process of obtaining nurse certification, identify resources available through the Lifespan Intranet and describe how certification aligns with Magnet Hospital designation.

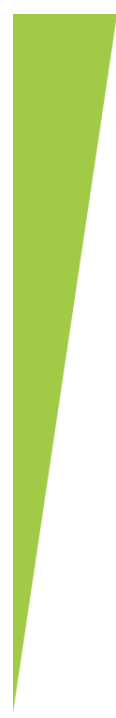

\section{Stroke Education for Nurses}

Module four 


\section{Objectives}

Describe process of obtaining Nurse certification

- Identify resources available through the Lifespan Intranet

- Describe how certification aligns with Magnet Hospital Designation

\section{Certification}

- "The mission of the American Nurses Credentialing Center (ANCC), a subsidiary of the American Nurses Association (ANA), is to promote excellence in nursing and health care globally through credentialing programs. ANCC's internationally renowned credentialing programs certify and recognize individual nurses in specialty practice areas; recognize healthcare organizations for promoting safe, positive work environments; and accredit continuing nursing education organizations."
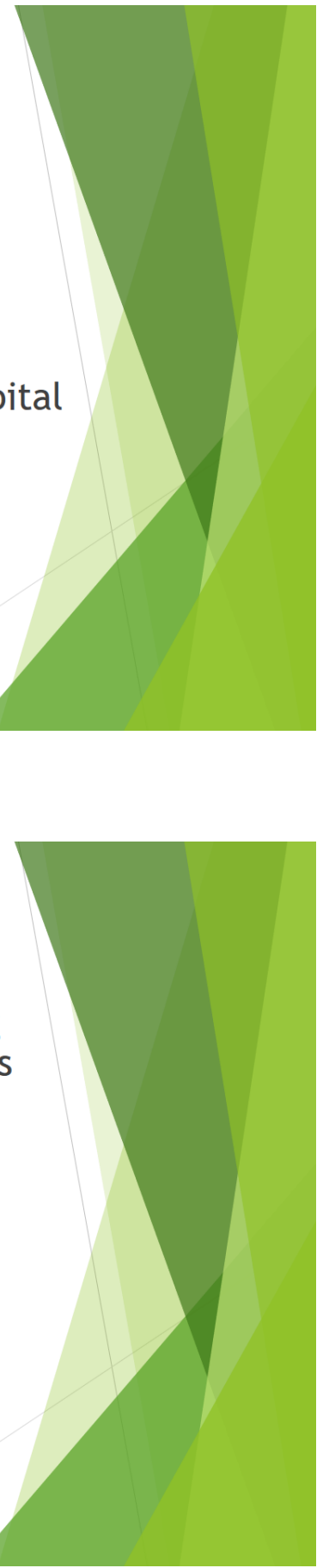


\section{American Nurses Credentialing Center (ANCC)}

http://www.nursecredentialing.org/default.aspx
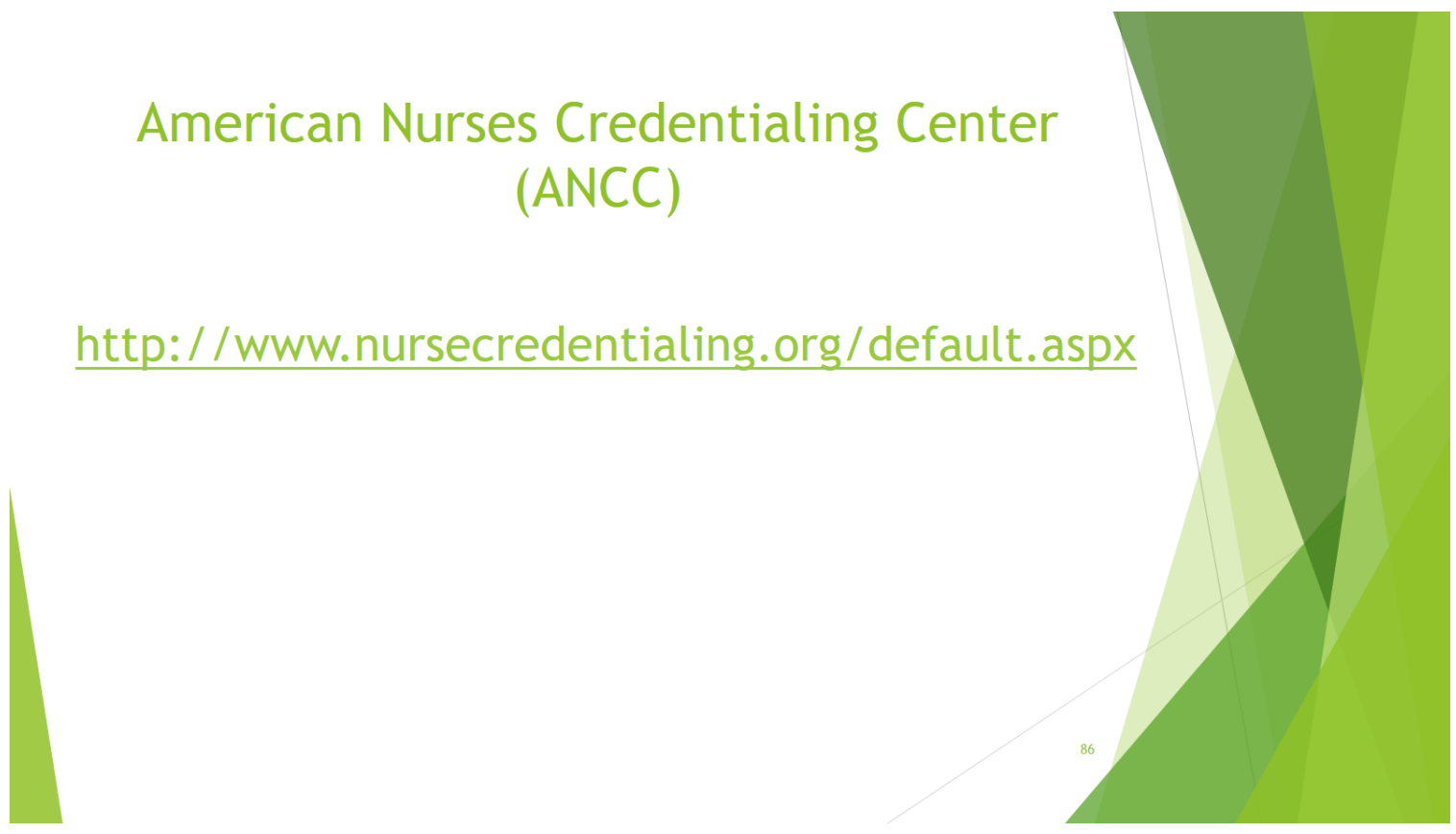

Lifespan Stroke Care Education Resources

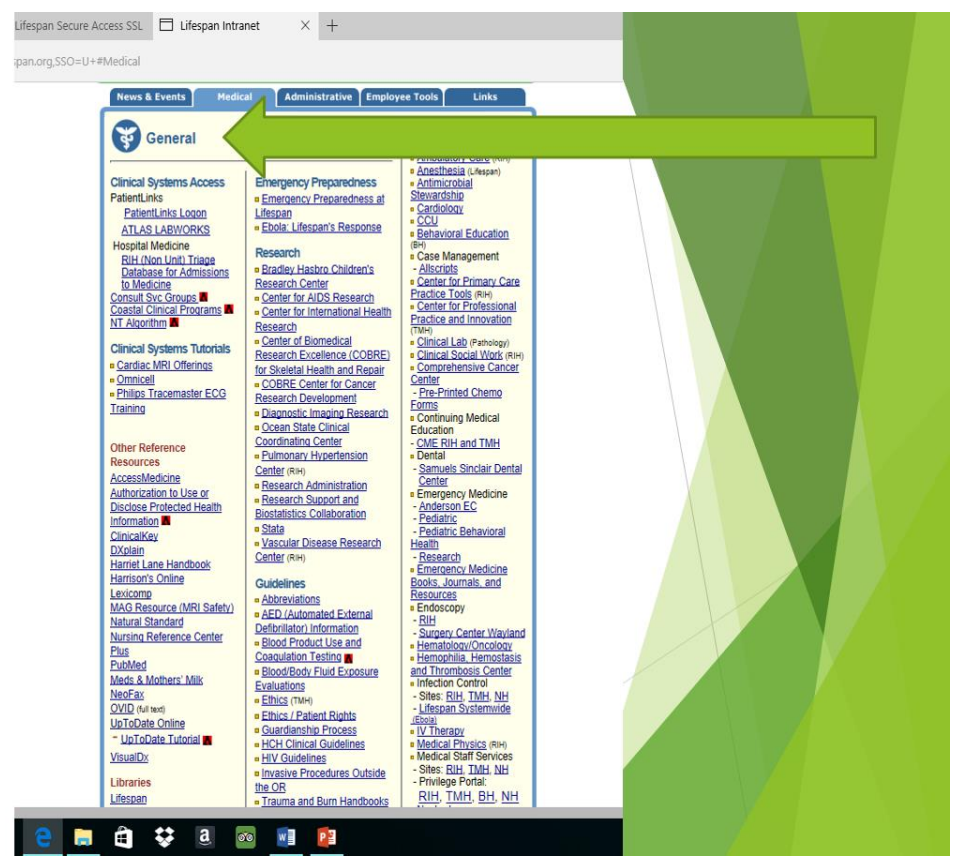




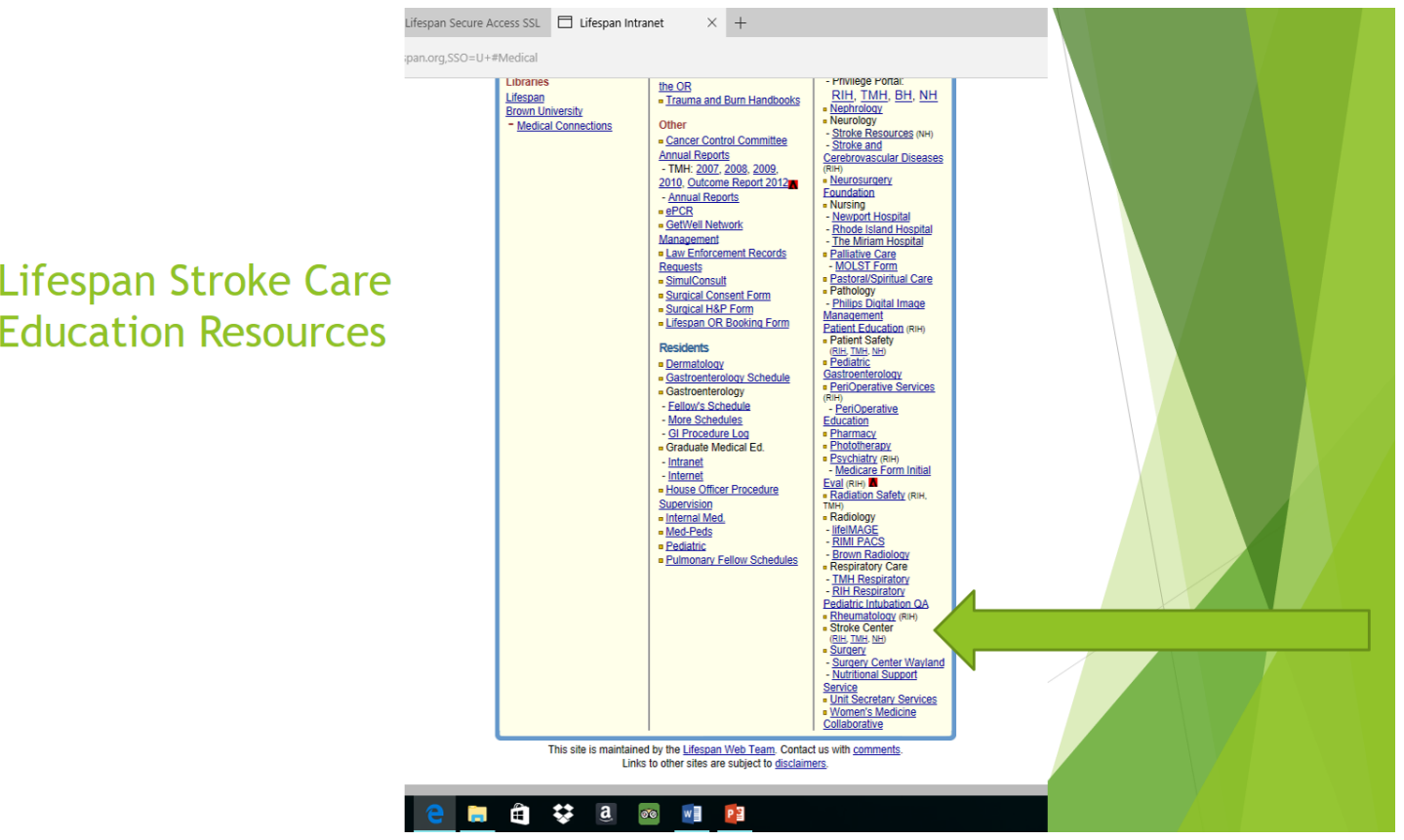

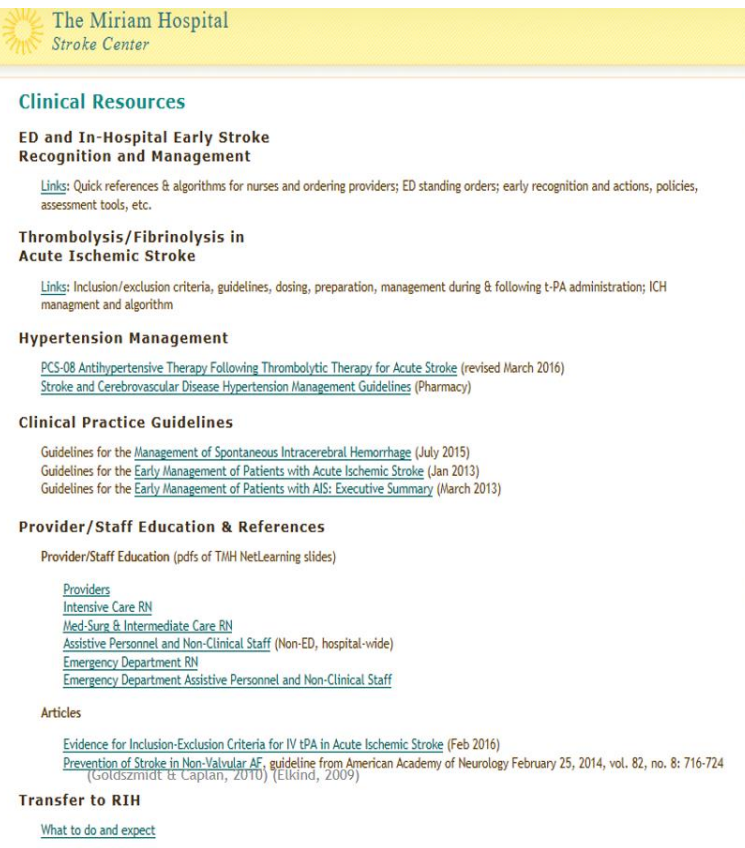

The Miriam Hospital Primary Stroke Center

Our Recognition for Quality

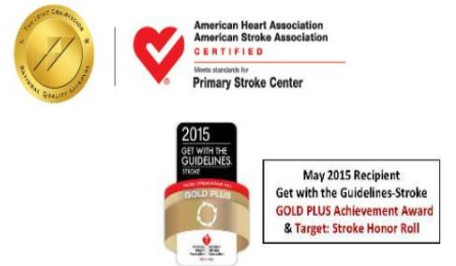

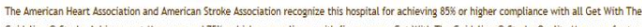

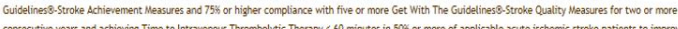

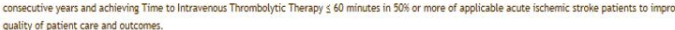
Our History

The Mirian Hosoital (TMH) was the first in Rhode Iland to be designated by The Joint Commission as a Primary Stroke Center. Our Mission

The Mirian Hospital (TMH) shall provide the highest quality stroke care, while working with the community and Emergency Medical
Services ENSS in fhode sland to improve issues affecting the rapid recognition and response for those with suspected stroke.

A.71 Stroke Center Pefformance Improvement Plan

What to do and expect 


\section{Additional Resources}

http: / / www.heart.org/HEARTORG /

https: / / www. jointcommission.org/

http: //www.americanbraincoalition.org/

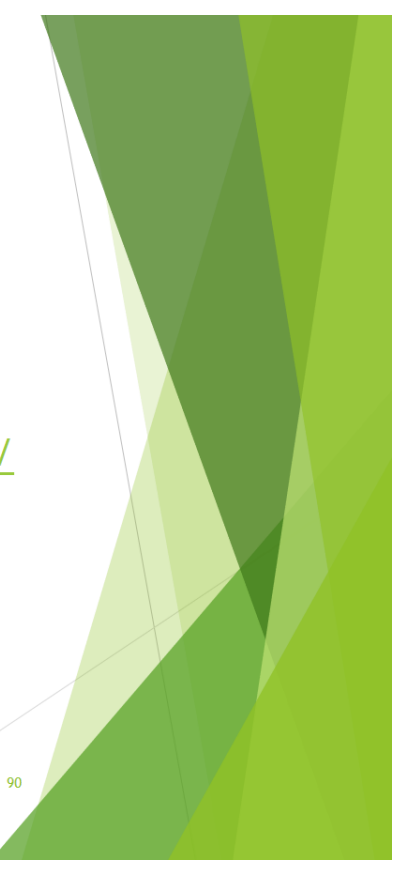

\section{References}

American Heart Association. (2016). Retrieved May 18, 2016, from American Heart Association: http://brainhealth.strokeassociation.org/

Davis, C. \& Lockhart, L. (2016). Update: Stroke. Nursing Management, 24-33.

Curley, M. (2007). Synergy: The unique relationship between nurses and patients. Indianapolis: Sigma Theta Tau International.

Jauch, E. C., Saver, J. L., Adams, H. P., Bruno, A., Demaerschalk, B. M., Khatri, P., ... \& Summers, D. R. (2013). Guidelines for the early management of patients with acute ischemic stroke a guideline for healthcare professionals from the American Heart Association/American Stroke Association. Stroke, 44(3), 870-947.

Tu, A. (2015). FDA information on medication errors involving Activase and TNKase. News for Health Professsionals.

Shulkin, D., Jewell, K., Alexandrov, A.W., Bernard, D., Brophy, G., Hess,

D.,....Kohlbrenner, J. (2011). Impact of systems of care and blood pressure

management on stroke outcomes. Population Health Management, 14, 267 -

275. Doi:10.1089/pop.2010.0068

The Joint Commission. (2016). Retrieved March, 12, 2016 from The Joint Commission: https://www. jointcommission.org/stroke/ 
